\title{
POSITIVE ENERGY QUANTIZATION OF LINEAR DYNAMICS
}

\author{
JAN DEREZIŃSKI \\ Department of Mathematical Methods in Physics, Warsaw University \\ Hoża 74, 00-682 Warszawa, Poland \\ E-mail: jan.derezinski@fuw.edu.pl \\ CHRISTIAN GÉRARD \\ Département de Mathématiques, Université de Paris Sud \\ 91405 Orsay Cedex, France \\ E-mail: christian.gerard@math.u-psud.fr
}

\begin{abstract}
The abstract mathematical structure behind the positive energy quantization of linear classical systems is described. It is separated into three stages: the description of a classical system, the algebraic quantization and the Hilbert space quantization. Four kinds of systems are distinguished: neutral bosonic, neutral bosonic, charged bosonic and charged fermionic.

The formalism that is described follows closely the usual constructions employed in quantum physics to introduce noninteracting quantum fields.
\end{abstract}

\section{Contents}

1. Introduction . . . . . . . . . . . . . . . . . . . 76

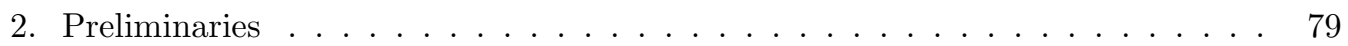

2.1. Vector spaces . . . . . . . . . . . . . . . . . . . 79

2.2. Symmetric forms . . . . . . . . . . . . . . . . . . . . . . . 79

2.3. Euclidean spaces . . . . . . . . . . . . . . . . . . . . 79

2.4. Symplectic spaces . . . . . . . . . . . . . . . . . . . . . . . . . 80

2.5. Sesquilinear forms . . . . . . . . . . . . . . . . . 80

2.6. Unitary spaces . . . . . . . . . . . . . . . . . . . 80

2.7. Charged symplectic spaces . . . . . . . . . . . . . . . 80

2000 Mathematics Subject Classification: 81T05.

Key words and phrases: canonical commutation relations, canonical anticommutation relations, Fock spaces, quantum field theory.

The paper is in final form and no version of it will be published elsewhere. 
2.8. Complexification of a vector space . . . . . . . . . . . . . . . . 81

2.9. Anti-involutions . . . . . . . . . . . . . . . . . . . . . 81

2.10. (Pseudo-)Kähler spaces . . . . . . . . . . . . . . . . . 81

2.11. U(1) symmetries of charge 1. . . . . . . . . . . . . . . . . 82

2.12. Operators on Hilbert spaces . . . . . . . . . . . . . . . . . . . . . . 82

3. Canonical commutation and anticommutation relations . . . . . . . . . . . 83

3.1. CCR representations . . . . . . . . . . . . . . . . 83

3.2. Regular CCR representations . . . . . . . . . . . . . . . . . . 83

3.3. Charged CCR representations . . . . . . . . . . . . . . . . . . . . . . . . . . . . . 84

3.4. CAR representations . . . . . . . . . . . . . . . . . . . 85

3.5. Charged CAR representations . . . . . . . . . . . . . . . . . 86

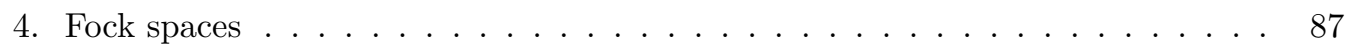

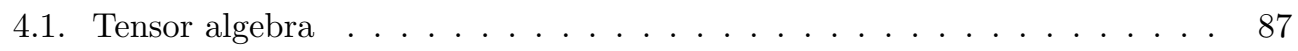

4.2. Operators $\mathrm{d} \Gamma$ and $\Gamma$ on the tensor algebra . . . . . . . . . . . . . . . 87

4.3. Bosonic and fermionic Fock spaces . . . . . . . . . . . . . . . . . . 88

4.4. $\mathrm{d} \Gamma$ and $\Gamma$ operators on Fock spaces . . . . . . . . . . . . . . . . . . . . . . 88

4.5. Creation and annihilation operators . . . . . . . . . . . . . . 88

4.6. Fock CCR representation . . . . . . . . . . . . . . . . . . . . . 89

4.7. Fock CAR representation . . . . . . . . . . . . . . . . . . . 89

$5 . \quad$ CCR and CAR algebras . . . . . . . . . . . . . . . . . . . . . . . . 89

5.1. Weyl CCR algebras . . . . . . . . . . . . . . . . . . . . . 9 90

5.2. Stone-von Neumann CCR algebras . . . . . . . . . . . . . . . . . . . 90

5.3. Regular CCR algebras . . . . . . . . . . . . . . . . . . . . . . . . . . . . . . . . . . 91

5.4. $C^{*}$-CAR algebras . . . . . . . . . . . . . . . . . . . . . . . . . . . . . . . . 92

6. Quantization of neutral systems . . . . . . . . . . . . . . . . . . . . . . . . . . . . 93

6.1. Neutral bosonic systems . . . . . . . . . . . . . . . . . . . 93

6.2. Neutral fermionic systems . . . . . . . . . . . . . . . . . . . . . . . . . . . . . . 96

6.3. Time reversal in neutral systems . . . . . . . . . . . . . . . . . . 97

7. Quantization of charged systems . . . . . . . . . . . . . . . . . . . 97

7.1. Charged bosonic systems . . . . . . . . . . . . . . . . . . . . . 98

7.2. Charged fermionic systems . . . . . . . . . . . . . . . . . . . 100

7.3. Charge reversal . . . . . . . . . . . . . . . . . . . . . . . . . . . . . . . . . . . . . . . . . . .

7.4. Time reversal in charged systems . . . . . . . . . . . . . . . . . . . 102

1. Introduction. In physics, by quantization one means various procedures that lead from classical systems to quantum systems. In this paper we describe the basic mathematical structure of the positive energy quantization of linear classical systems. One of its basic requirements is the implementation of the quantum dynamics by a positive Hamiltonian. In all the cases we consider, the resulting Hilbert space has a natural structure of a Fock space, and the dynamics is obtained by the so-called second quantization of the dynamics on the 1-particle space.

Linear classical systems that we have in mind often have an infinite number of degrees of freedom. The most typical examples are the space of solutions of the Klein-Gordon and 
of the Dirac equation, possibly on a curved space-time and in the presence of external potentials. We can also consider other systems, not necessarily relativistic, e.g. motivated by the condensed matter physics at zero temperature.

The positive energy quantization, which we describe in an abstract fashion in this paper, is used in quantum field theory as the starting point for the construction of free (that means noninteracting) quantum fields and many-body quantum systems. We will not discuss the quantization of nonlinear dynamics, which is usually more difficult and often ambiguous.

In quantum physics, one can distinguish two basic types of particles: bosons and fermions.

Classical theories describing bosons have a natural symplectic structure. After quantization, one obtains quantum fields satisfying canonical commutation relations (abbreviated CCR). They are usually represented on a bosonic Fock space.

Classical theories describing fermions possess a natural euclidean structure. The corresponding quantum fields satisfy canonical anticommutation relations (abbreviated CAR). They are usually represented on a fermionic Fock space.

Thus classical bosonic and fermionic systems equipped with a linear dynamics can be described by a symplectic, resp. euclidean vector space $\mathcal{Y}$. This space has the interpretation of the dual of the classical phase space. (We will not be very pedantic about this point, and we will usually call $\mathcal{Y}$ the phase space as well). The classical dynamics is described by a 1-parameter group $\mathbb{R} \ni t \mapsto r_{t}$ of linear symplectic, resp. orthogonal transformations on $\mathcal{Y}$.

Both fermionic and bosonic systems appear in two varieties: neutral and charged. In the case of charged systems, the phase space is in addition endowed with an action of the group $U(1)$. Assuming that it is a representation of charge 1, it is natural to encode this symmetry by viewing the phase space as a complex vector space. In the case of neutral systems, the space $\mathcal{Y}$ is assumed to be real.

More precisely, on the classical level charged bosonic systems are described by a complex vector space equipped with a nondegenerate antihermitian form. We will call such spaces charged symplectic. We will assume that the dynamics $r_{t}$ preserves this form.

Charged fermionic systems are described by a unitary space (complex space equipped with a positive scalar product) and its dynamics is a 1-parameter unitary group.

To sum up, we distinguish 4 basic formalisms for quantization:

(1) Neutral bosonic formalism, applied e.g. to real solutions of the Klein-Gordon equation;

(2) Neutral fermionic formalism, applied e.g. to Majorana spinors satisfying the Dirac equation;

(3) Charged bosonic systems, applied e.g. to complex solutions of the Klein-Gordon equation;

(4) Charged fermionic systems, applied e.g. to Dirac spinors satisfying the Dirac equation.

REMARK 1.1. Note that in the most common physics applications one uses the neutral bosonic formalism (e.g. for photons) and the charged fermionic formalism (e.g. for elec- 
trons). Charged bosons are also quite common, e.g. charged pions or gauge bosons in the standard model. On the other hand, until recently, the neutral fermionic formalism had mostly theoretical interest. However, in the modern version of the standard model right-handed massive neutrinos are neutral fermions described by Majorana spinors $[\mathrm{Sr}]$.

One can distinguish 3 stages of quantization:

(1) Classical system. We consider one of the four kinds of the phase space $\mathcal{Y}$, together with a 1-parameter group of its automorphisms, $\mathbb{R} \ni t \mapsto r_{t}$, which we view as a classical dynamics.

(2) Algebraic quantization. We choose an appropriate $*$-algebra $\mathfrak{A}$, together with a 1-parameter group of $*$-automorphisms $\mathbb{R} \ni t \mapsto \hat{r}_{t}$. The algebra $\mathfrak{A}$ is sometimes called the field algebra of the quantum system. The commutation, resp. anticommutation relations satisfied by the appropriate distinguished elements of $\mathfrak{A}$ are dictated by the additional structure of the phase space, e.g. symplectic, euclidean, etc. The 1-parameter group of $*$-automorphisms $\hat{r}_{t}$ describes the quantum dynamics in the Heisenberg picture. The algebra $\mathfrak{A}$ contains operators that are useful in the theoretical description of the system. However, we do not assume that all of its elements are physically observable, even in principle. Therefore, we also distinguish the algebra of observables, which is a certain subalgebra of $\mathfrak{A}$, invariant with respect to the dynamics, that consists of operators whose measurement is theoretically possible.

(3) Hilbert space quantization. We represent the algebra $\mathfrak{A}$ on a certain Hilbert space $\mathcal{H}$, so that the dynamics is implemented by a 1-parameter unitary group generated by a positive operator $H$, called the Hamiltonian. Typically, this representation is faithful, so that we can write $\mathfrak{A} \subset B(\mathcal{H})$ and

$$
\hat{r}_{t}(A)=\mathrm{e}^{\mathrm{i} t H} A \mathrm{e}^{-\mathrm{i} t H} .
$$

The description of quantization that one can find in numerous textbooks on quantum field theory is almost always presented in a certain concrete context, typically that of the Klein-Gordon or Dirac equation. In our approach we describe only the abstract underlying mathematical structure. Let us stress, however, that our presentation, in spite of its abstract mathematical language, follows very closely the usual exposition, see e.g. We], and $[\mathrm{Sr}$; in particular $[\mathrm{Sr}]$ Sec. 22 for complex bosons and $[\mathrm{Sr}] \mathrm{Sec} .49$ for neutral fermions.

Note that among the three stages of quantization described above, the most important is the first and the third. The second stage - the algebraic quantization - can be skipped altogether. In the usual presentation, typical for physics textbooks, it is limited to a formal level - one says that "commuting classical observables" are replaced by "noncommuting quantum observables" satisfying the appropriate commutation, resp. anticommutation relations. In our presentation, we tried to interpret this statement in terms of well defined $C^{*}$-algebras. This is quite easy in the case of fermions. Unfortunately, in the case of bosons it leads to certain technical difficulties related to the unboundedness of bosonic fields. We discuss a number of possible choices for $C^{*}$-algebras describing bosonic observables. To some extent, the algebraic quantization is merely an exercise of academic interest. Nevertheless, in some situations it sheds light on some conceptual aspects of quantum theory. 
One of the confusing conceptual points that we believe our abstract approach can explain is the difference between the phase space and the 1-particle space. Throughout our paper, the former is typically denoted by $\mathcal{Y}$ and the latter by $\mathcal{Z}$. These two spaces are often identified. They have, however, a different physical meaning and are equipped with a different algebraic structure.

We also discuss abstract properties of two commonly used discrete symmetries of quantum systems: the time reversal and the charge reversal. Their properties can be quite confusing. We believe that the precise language of linear algebra is particularly adapted to explain their properties. Note, for instance, that the charge reversal is antilinear with respect to the complex structure on the phase space and linear with respect to the complex structure on the 1-particle space. On the other hand, the time reversal is antilinear with respect to both.

Among well-known textbooks about rigorous foundations of quantum field theory one can quote [BSZ, BR, Em, GJ, Ha, RS2, Si]. In particular, [BSZ] contains a discussion of some of the aspects of the positive energy quantization. One can argue that our paper collects some elements of the knowledge that belongs to the folklore of theoretical and mathematical physics. Nevertheless, a systematic and comprehensive discusssion of the topic described in our paper is to our knowledge difficult to find in the literature. We are preparing a monograph DG2 that will cover it in greater detail.

2. Preliminaries. In this section we introduce a precise terminology and notation, mostly about linear algebra, which we will use in this paper. All of this section is very elementary. A large part of it is standard and well-known. For some concepts we found it convenient to invent new names. The reader may wonder why we need to be so pedantic. We will see later on that a surprisingly large variety of concepts from basic linear algebra plays an important role in quantization.

2.1. Vector spaces. Let $\mathcal{Y}, \mathcal{W}$ be vector spaces over the field $\mathbb{K}=\mathbb{R}$ or $\mathbb{K}=\mathbb{C} . L(\mathcal{Y}, \mathcal{W})$ denotes the space of linear maps from $\mathcal{Y}$ to $\mathcal{W}$.

If $\mathcal{Y}$ is a complex space, then $\mathcal{Y}_{\mathbb{R}}$ will denote its real form, that is $\mathcal{Y}$ considered as a real space.

2.2. Symmetric forms. Let $\mathcal{Y}$ be a vector space. Consider a bilinear form $\nu$ on $\mathcal{Y}$

$$
\mathcal{Y} \times \mathcal{Y} \ni\left(y_{1}, y_{2}\right) \mapsto y_{1} \nu y_{2} \in \mathbb{K}
$$

We will say that $\nu$ is symmetric if

$$
y_{1} \nu y_{2}=y_{2} \nu y_{1}, \quad y_{1}, y_{2} \in \mathcal{Y} .
$$

2.3. Euclidean spaces. Let $\nu$ be a symmetric form on a real space $\mathcal{Y}$. It is called positive if $y \nu y>0$ for $y \neq 0$. A couple $(\mathcal{Y}, \nu)$, where $\nu$ is a positive form, is called a euclidean space. If $\mathcal{Y}$ is complete for the norm $\|y\|:=\sqrt{y \nu y}$, then it is called a real Hilbert space.

Let $(\mathcal{Y}, \nu)$ be a euclidean space and $r \in L(\mathcal{Y})$. We say that $r$ is orthogonal if $r$ is bijective and $\left(r y_{1}\right) \nu r y_{2}=y_{1} \nu y_{2}$. 
The set of orthogonal elements in $L(\mathcal{Y})$ is a group for the operator composition, denoted by $O(\mathcal{Y})$.

If the form $\nu$ is nondegenerate, but not necessarily positive, then all the definitions are the same except that we add the prefix "pseudo-" to the words "euclidean", "isometric" and "orthogonal".

2.4. Symplectic spaces. Let $\mathcal{Y}$ be a vector space. We say that a bilinear form $\omega$

$$
\mathcal{Y} \times \mathcal{Y} \ni\left(y_{1}, y_{2}\right) \mapsto y_{1} \omega y_{2} \in \mathbb{K}
$$

is antisymmetric if

$$
y_{1} \omega y_{2}=-y_{2} \omega y_{1}, \quad y_{1}, y_{2} \in \mathcal{Y} \text {. }
$$

A nondegenerate antisymmetric bilinear form is called a symplectic form. The pair $(\mathcal{Y}, \omega)$ is then called a symplectic space.

Let $(\mathcal{Y}, \omega)$ be a symplectic space and $r \in L(\mathcal{Y})$. We say that

$r$ is symplectic if $r$ is bijective and $\left(r y_{1}\right) \omega r y_{2}=y_{1} \omega y_{2}$,

$r$ is antisymplectic if $r$ is bijective and $\left(r y_{1}\right) \omega r y_{2}=-y_{1} \omega y_{2}$.

2.5. Sesquilinear forms. Let $\mathcal{Z}$ be a complex vector space. Consider a sesquilinear form (antilinear in the first argument, linear in the second)

$$
\mathcal{Z} \times \mathcal{Z} \ni\left(z_{1}, z_{2}\right) \mapsto\left(z_{1} \mid \beta z_{2}\right) \in \mathbb{C}
$$

We say that

$$
\begin{aligned}
\beta \text { is hermitian if }\left(z_{2} \mid \beta z_{1}\right) & =\overline{\left(z_{1} \mid \beta z_{2}\right)}, z_{1}, z_{2} \in \mathcal{Z}, \\
\beta \text { is antihermitian } & \text { if }\left(z_{2} \mid \beta z_{1}\right)=-\overline{\left(z_{1} \mid \beta z_{2}\right)}, z_{1}, z_{2} \in \mathcal{Z},
\end{aligned}
$$

Clearly, $\beta$ is hermitian iff i $\beta$ is antihermitian.

2.6. Unitary spaces. A hermitian form $\beta$ is called positive if $(z \mid \beta z)>0$ for $z \neq 0$. It is often called a scalar product. A pair $(\mathcal{Z},(\cdot \mid \beta \cdot))$ is then sometimes called a unitary space. If $\mathcal{Z}$ is complete for the norm $\|z\|:=\sqrt{(z \mid \beta z)}$, then it is called a Hilbert space.

Let $r \in L(\mathcal{Z})$.

$$
r \text { is unitary if } r \text { is bijective and }\left(r z_{1} \mid \beta r z_{2}\right)=\left(z_{1} \mid \beta z_{2}\right) .
$$

The set of unitary operators on $\mathcal{Z}$ is a group denoted by $U(\mathcal{Z})$.

Let $r \in L\left(\mathcal{Z}_{\mathbb{R}}\right)$ be antilinear. We say that

$$
r \text { is antiunitary if } r \text { is bijective and }\left(r z_{1} \mid \beta r z_{2}\right)=\overline{\left(z_{1} \mid \beta z_{2}\right)} \text {. }
$$

2.7. Charged symplectic spaces. If $\omega$ is antihermitian and nondegenerate, then $(\mathcal{Z}, \omega)$ is called a charged symplectic space.

Let $(\mathcal{Z}, \omega)$ be a charged symplectic space and $r \in L(\mathcal{Z})$. We say that

$r$ is charged symplectic if $r$ is bijective and $\left(r z_{1} \mid \omega r z_{2}\right)=\left(z_{1} \mid \omega z_{2}\right)$,

$r$ is charged antisymplectic if $r$ is bijective and $\left(r z_{1} \mid \omega r z_{2}\right)=-\left(z_{1} \mid \omega z_{2}\right)$.

The set of charged symplectic operators on $\mathcal{Z}$ is a group for the operator composition denoted by $C h S p(\mathcal{Z})$. 
Let $r \in L\left(\mathcal{Z}_{\mathbb{R}}\right)$ be antilinear. We adopt the following terminology for various kinds of antilinear operators on a charged symplectic space:

$r$ is anticharged symplectic if $r$ is bijective and $\left(z_{1} \mid \omega z_{2}\right)=\overline{\left(r z_{1} \mid \omega r z_{2}\right)}$,

$r$ is anticharged antisymplectic if $r$ is bijective and $\left(z_{1} \mid \omega z_{2}\right)=-\overline{\left(r z_{1} \mid \omega r z_{2}\right)}$.

REMARK 2.1. The terminology "charged symplectic space" is motivated by applications in quantum field theory: such spaces describe charged bosons.

2.8. Complexification of a vector space. Consider a real space $\mathcal{Y}$. Let $\mathbb{C} \mathcal{Y}$ denote its complexification, that is $\mathbb{C} \otimes_{\mathbb{R}} \mathcal{Y}$, which is a complex vector space equipped with a natural conjugation denoted by $\mathbb{C} \mathcal{Y} \ni w \mapsto \bar{w} \in \mathbb{C} \mathcal{Y}$.

Clearly, every $r \in L(\mathcal{Y})$ extends uniquely to a linear operator on $\mathbb{C} \mathcal{Y}$, which will be denoted $r_{\mathbb{C}}$ and to a unique antilinear operator on $\mathbb{C} \mathcal{Y}$, which will be denoted $r_{\overline{\mathbb{C}}}$.

2.9. Anti-involutions. Let $\mathcal{Y}$ be a vector space. We say that $\mathrm{j} \in L(\mathcal{Y})$ is an antiinvolution, if $\mathrm{j}^{2}=-1$.

Let $\mathcal{Y}$ be a real space equipped with an anti-involution $\mathrm{j} \in L(\mathcal{Y})$. We can consider it as a complex space, with $\mathrm{j}$ identified with the imaginary unit $\mathrm{i}$, and then we will denote it by $\mathcal{Y}^{\mathbb{C}}$. However, we will seldom do so, and in what follows we treat $\mathcal{Y}$ as a real space.

Note that $(\mathbb{C Y})_{\mathbb{R}}$ has two distinguished anti-involutions: the usual i, and also $\mathrm{j}_{\mathbb{C}}$. Set

$$
\mathcal{Z}:=\left\{y-\mathrm{ij}_{\mathbb{C}} y: y \in \mathcal{Y}\right\}, \quad \overline{\mathcal{Z}}:=\left\{y+\mathrm{ij}_{\mathbb{C}} y: y \in \mathcal{Y}\right\} .
$$

$\mathcal{Z}$ will be called the holomorphic subspace of $\mathbb{C} \mathcal{Y}, \overline{\mathcal{Z}}$ will be called the antiholomorphic subspace of $\mathbb{C} \mathcal{Y}$. The corresponding projections equal $\mathbb{1}_{\mathcal{Z}}:=\frac{1}{2}\left(\mathbb{1}-\mathrm{ij}_{\mathbb{C}}\right)$ and $\mathbb{1}_{\overline{\mathcal{Z}}}:=\frac{1}{2}\left(\mathbb{1}+\mathrm{ij}_{\mathbb{C}}\right)$. Clearly, $\mathbb{1}=\mathbb{1}_{\mathcal{Z}}+\mathbb{1}_{\overline{\mathcal{Z}}}$, and $\mathbb{C} \mathcal{Y}=\mathcal{Z} \oplus \overline{\mathcal{Z}}$. We have $\mathcal{Z}=\operatorname{Ker}\left(\mathrm{j}_{\mathbb{C}}-\mathrm{i}\right), \overline{\mathcal{Z}}=\operatorname{Ker}\left(\mathrm{j}_{\mathbb{C}}+\mathrm{i}\right)$.

The converse construction is as follows: Let $\mathcal{Z}$ be a complex vector space. Let $\overline{\mathcal{Z}}$ be the space complex conjugate to $\mathcal{Z}$ (naturally isomorphic to $\mathcal{Z}$ as a real space, but with the opposite complex structure). Set

$$
\mathcal{Y}:=\operatorname{Re}(\mathcal{Z} \oplus \overline{\mathcal{Z}}):=\{(z, \bar{z}) \in \mathcal{Z} \oplus \overline{\mathcal{Z}}: z \in \mathcal{Z}\} .
$$

Clearly $\mathcal{Y}$ is a real vector space equipped with the anti-involution

$$
\mathrm{j}(z, \bar{z}):=(\mathrm{i} z, \overline{\mathrm{i} z})=(\mathrm{i} z,-\mathrm{i} \bar{z}) .
$$

2.10. (Pseudo-)Kähler spaces. Let $(\cdot \mid \beta \cdot)$ be a hermitian form on a complex space $\mathcal{Y}$. Then on $\mathcal{Y}_{\mathbb{R}}$ we have a symmetric form $\nu$,

$$
y_{2} \nu y_{1}:=\operatorname{Re}\left(y_{2} \mid \beta y_{1}\right),
$$

an antisymmetric form $\omega$,

$$
y_{2} \omega y_{1}:=\operatorname{Im}\left(y_{2} \mid \beta y_{1}\right)
$$

and an anti-involution $\mathrm{j}$,

$$
\mathrm{j} y:=\mathrm{i} y \text {. }
$$

Note the relationship $y_{1} \omega \mathrm{j} y_{2}=y_{1} \nu y_{2}$. 
The name pseudo-Kähler space will be used for a space equipped with a nondegenerate hermitian form treated as a real space with the three structures 2.2 , 2.3. and 2.4. Below we give a more precise definition:

Definition 2.2. We say that a quadruple $(\mathcal{Y}, \nu, \omega, \mathrm{j})$ is a pseudo-Kähler space if

(1) $\mathcal{Y}$ is a real vector space,

(2) $\nu$ is a nondegenerate symmetric form,

(3) $\omega$ is a nondegenerate antisymmetric form,

(4) $\mathrm{j}$ is an anti-involution,

(5) $y_{1} \omega \mathrm{j} y_{2}=y_{1} \nu y_{2}, y_{1}, y_{2} \in \mathcal{Y}$.

If in addition $\nu$ is positive, then we say that $(\mathcal{Y}, \nu, \omega, \mathrm{j})$ is a Kähler space.

Two structures out of $\nu, \omega, \mathrm{j}$ determine the third. This motivates the following definitions:

DeFINITION 2.3 .

(1) Let $(\mathcal{Y}, \omega)$ be a symplectic space. We say that an anti-involution $\mathrm{j}$ is pseudo-Kähler if $y_{1} \omega \mathrm{j} y_{2}$ is a symmetric form. If in addition it is positive, then we say that $\mathrm{j}$ is Kähler.

(2) Let $(\mathcal{Y}, \nu)$ be a euclidean space. We say that an anti-involution $\mathrm{j}$ is Kähler if $y_{1} \nu \mathrm{j} y_{2}$ is an antisymmetric form.

The definitions (1) and (2) have other equivalent versions, as seen from the following theorem:

THEOREM 2.4 .

(1) Let $(\mathcal{Y}, \omega)$ be a symplectic space. Then $(\omega, \mathrm{j})$ pseudo-Kähler iff $\mathrm{j} \in S p(\mathcal{Y})$.

(2) Let $(\mathcal{Y}, \nu)$ be a euclidean space. Then $(\nu, \mathrm{j})$ is Kähler iff $\mathrm{j} \in O(\mathcal{Y})$.

2.11. $U(1)$ symmetries of charge 1 . Let $\mathcal{Y}$ be a real space. Let $U(1)$ be the group $\mathbb{R} / 2 \pi \mathbb{Z}$. Let $U(1) \ni \theta \mapsto u_{\theta} \in L(\mathcal{Y})$ be a representation.

Definition 2.5. We say that it is a representation of charge 1 if there exists an antiinvolution $\mathrm{j}_{\mathrm{ch}}$ such that

$$
u_{\theta}=\cos \theta \mathbb{1 1}+\sin \theta \mathrm{j}_{\mathrm{ch}} .
$$

Proposition 2.6. Let $\left(u_{\theta}\right)_{\theta \in U(1)}$ be a representation of charge 1 .

(1) Assume that $\mathcal{Y}$ is a symplectic space. Then $u_{\theta}$ is symplectic for $\theta \in U(1)$ iff $\mathrm{j}_{\mathrm{ch}}$ is pseudo-Kähler.

(2) Assume that $\mathcal{Y}$ is a euclidean space. Then $u_{\theta}$ is orthogonal for $\theta \in U(1)$ iff $\mathrm{j}_{\mathrm{ch}}$ is Kähler.

2.12. Operators on Hilbert spaces. If $\mathcal{H}, \mathcal{K}$ are Hilbert spaces, then $B(\mathcal{H}, \mathcal{K})$, $U(\mathcal{H}, \mathcal{K})$, resp. $C l(\mathcal{H}, \mathcal{K})$ denotes the space of bounded, unitary, resp. closed operators from $\mathcal{H}$ to $\mathcal{K}$. We set $B(\mathcal{H}):=B(\mathcal{H}, \mathcal{H}), U(\mathcal{H}):=U(\mathcal{H}, \mathcal{H}), C l(\mathcal{H}):=C l(\mathcal{H}, \mathcal{H})$.

$B_{\mathrm{h}}(\mathcal{H})$, resp. $C l_{\mathrm{h}}(\mathcal{H})$ denotes the set of bounded selfadjoint, resp. closed selfadjoint operators on $\mathcal{H}$.

If $\left(\mathcal{Z}_{i}\right)_{i \in I}$ is a family of Hilbert spaces, then $\oplus_{i \in I} \mathcal{Z}_{i}$ will always denote the direct sum in the sense of Hilbert spaces. 
If $\mathcal{Z}, \mathcal{W}$ are Hilbert spaces, then $\mathcal{Z} \otimes \mathcal{W}$ will always denote the tensor product of $\mathcal{Z}$ and $\mathcal{W}$ in the sense of Hilbert spaces.

3. Canonical commutation and anticommutation relations. In this section first we will discuss the concept of a representation of canonical commutation relations, abbreviated a CCR representation. Then we will introduce the notion of a representation of canonical anticommutation relations, abbreviated a CAR representation. Both concepts come in two varieties: neutral and charged.

CCR and CAR representations have a long history, going back to e.g. [Di1, JW]. They were for quite some time an important subject of research in mathematical physics, let us mension [Ar1, Ar2, Ar3, ArShi, CMR, Sla]. For a textbook reference on CCR and CAR representations let us quote [BR, see also De].

Throughout this section, $\mathcal{H}$ will denote a Hilbert space.

3.1. CCR representations. Let $(\mathcal{Y}, \omega)$ be a symplectic space. Let us first try to define the concept of a CCR representation in a naive way. We would like to have a linear map

$$
\mathcal{Y} \ni y \mapsto \phi^{\pi}(y) \in C l_{\mathrm{h}}(\mathcal{H})
$$

satisfying

$$
\left[\phi^{\pi}\left(y_{1}\right), \phi^{\pi}\left(y_{2}\right)\right]=\mathrm{i} y_{1} \omega y_{2} \mathbb{1} .
$$

We will call 3.2 the canonical commutation relation in the Heisenberg form. Unfortunately, this relation is problematic from the rigorous point of view, because one needs to supply it with the precise meaning of the commutator of unbounded operators on the left hand side.

Weyl proposed to replace 3.2 with another relation involving the operators $\mathrm{e}^{\mathrm{i} \phi^{\pi}(y)}$. These operators are bounded, and therefore one does not need to discuss domain questions. In our definition of CCR representations we will use the canonical commutation relations in the so-called Weyl form. Under additional regularity assumptions they imply the CCR in the Heisenberg form.

DEFINITION 3.1. A representation of the canonical commutation relations or a $C C R$ representation over $(\mathcal{Y}, \omega)$ in $\mathcal{H}$ is a map

$$
\mathcal{Y} \ni y \mapsto W^{\pi}(y) \in U(\mathcal{H})
$$

satisfying

$$
W^{\pi}\left(y_{1}\right) W^{\pi}\left(y_{2}\right)=\mathrm{e}^{-\frac{\mathrm{i}}{2} y_{1} \omega y_{2}} W^{\pi}\left(y_{1}+y_{2}\right) .
$$

$W^{\pi}(y)$ is then called the Weyl operator corresponding to $y \in \mathcal{Y}$.

\subsection{Regular CCR representations}

Definition 3.2. A CCR representation (3.3) is called regular if

$$
\mathbb{R} \ni t \mapsto W^{\pi}(t y) \in U(\mathcal{H}) \quad \text { is strongly continuous for any } y \in \mathcal{Y} \text {. }
$$

Clearly, $\mathbb{R} \ni t \mapsto W^{\pi}(t y)$ is a strongly continuous 1-parameter unitary group. By the Stone theorem, for any $y \in \mathcal{Y}$, we can define its selfadjoint generator

In other words, $\mathrm{e}^{\mathrm{i} \phi^{\pi}(y)}=W^{\pi}(y)$.

$$
\phi^{\pi}(y):=-\left.\mathrm{i} \frac{\mathrm{d}}{\mathrm{d} t} W^{\pi}(t y)\right|_{t=0} .
$$


Definition 3.3. $\phi^{\pi}(y)$ will be called the (bosonic) field operator corresponding to $y \in \mathcal{Y}$.

Let $w \in \mathbb{C} \mathcal{Y}$. We can write $w=y_{1}+\mathrm{i} y_{2}$ for $y_{1}, y_{2} \in \mathcal{Y}$. We set

$$
\phi^{\pi}(w):=\phi^{\pi}\left(y_{1}\right)+\mathrm{i} \phi^{\pi}\left(y_{2}\right) .
$$

with $\operatorname{Dom} \phi^{\pi}(w):=\operatorname{Dom} \phi^{\pi}\left(y_{1}\right) \cap \operatorname{Dom} \phi^{\pi}\left(y_{2}\right)$.

Definition 3.4. $\phi^{\pi}(w)$ will be called the complex field operator corresponding to $w \in \mathbb{C} \mathcal{Y}$.

It is easy to show the following proposition:

Proposition 3.5. Let $y, y_{1}, y_{2} \in \mathcal{Y}$.

(1) $\phi^{\pi}(y)$ are closed.

(2) $\phi^{\pi}(t y)=t \phi^{\pi}(y), t \in \mathbb{R}$.

(3) On $\operatorname{Dom} \phi^{\pi}\left(y_{1}\right) \cap \operatorname{Dom} \phi^{\pi}\left(y_{2}\right)$ we have $\phi^{\pi}\left(y_{1}+y_{2}\right)=\phi^{\pi}\left(y_{1}\right)+\phi^{\pi}\left(y_{2}\right)$.

(4) $\left[\phi^{\pi}\left(y_{1}\right), \phi^{\pi}\left(y_{2}\right)\right]=\mathrm{i} y_{1} \omega y_{2} \mathbb{1}$ holds as a quadratic form on $\operatorname{Dom} \phi^{\pi}\left(y_{1}\right) \cap \operatorname{Dom} \phi^{\pi}\left(y_{2}\right)$.

3.3. Charged CCR representations. CCR representations, as defined in Def. 3.1. are used mainly to describe neutral bosons. Therefore, sometimes we will call them neutral CCR representations. In the context of charged bosons one uses another formalism described in the following definition.

Let $(\mathcal{Y},(\cdot \mid \omega \cdot))$ be a charged symplectic space. Let us first try to define a charged CCR representation in a naive way. We would like to have a linear map $\mathcal{Y} \ni y \mapsto \psi^{\pi}(y) \in C l(\mathcal{H})$ satisfying

$$
\begin{aligned}
{\left[\psi^{\pi *}\left(y_{1}\right), \psi^{\pi *}\left(y_{2}\right)\right] } & =\left[\psi^{\pi}\left(y_{1}\right), \psi^{\pi}\left(y_{2}\right)\right]=0, \\
{\left[\psi^{\pi}\left(y_{1}\right), \psi^{\pi *}\left(y_{2}\right)\right] } & =\mathrm{i}\left(y_{1} \mid \omega y_{2}\right) 1, \quad y_{1}, y_{2} \in \mathcal{Y} .
\end{aligned}
$$

Again, the above definition is problematic. A possible rigorous definition is given below:

Definition 3.6. We say that a map

$$
\mathcal{Y} \ni y \mapsto \psi^{\pi}(y) \in C l(\mathcal{H})
$$

is a charged $C C R$ representation iff there exists a map

$$
\mathcal{Y} \ni y \mapsto \phi^{\pi}(y) \in C l_{\mathrm{h}}(\mathcal{H})
$$

such that

$$
\mathrm{e}^{\mathrm{i} \phi^{\pi}\left(y_{1}\right)} \mathrm{e}^{\mathrm{i} \phi^{\pi}\left(y_{1}\right)}=\mathrm{e}^{-\frac{1}{2} \operatorname{Re}\left(y_{1} \mid \omega y_{2}\right)} \mathrm{e}^{\mathrm{i} \phi^{\pi}\left(y_{1}+y_{2}\right)}, y_{1}, y_{2} \in \mathcal{Y},
$$

$\phi^{\pi}(t y)=t \phi^{\pi}(y), t \in \mathbb{R}, \operatorname{Dom} \psi^{\pi}(y)=\operatorname{Dom} \phi^{\pi}(y) \cap \operatorname{Dom} \phi^{\pi}(\mathrm{i} y)$ and

$$
\psi^{\pi}(y)=\frac{1}{\sqrt{2}}\left(\phi^{\pi}(y)+\mathrm{i} \phi^{\pi}(\mathrm{i} y)\right), \quad y \in \mathcal{Y} .
$$

Note that a charged CCR representation satisfies the conditions of the "naive definition":

Proposition 3.7. Consider a charged CCR representation. Let $y, y_{1}, y_{2} \in \mathcal{Y}$.

(1) $\psi^{\pi}(\lambda y)=\lambda \psi^{\pi}(y), \lambda \in \mathbb{C}$;

(2) On $\operatorname{Dom} \psi^{\pi}\left(y_{1}\right) \cap \operatorname{Dom} \psi^{\pi}\left(y_{2}\right)$ we have $\psi^{\pi}\left(y_{1}+y_{2}\right)=\psi^{\pi}\left(y_{1}\right)+\psi^{\pi}\left(y_{2}\right)$; 
(3) In the sense of quadratic forms, we have the identities

$$
\begin{aligned}
{\left[\psi^{\pi *}\left(y_{1}\right), \psi^{\pi *}\left(y_{2}\right)\right] } & =\left[\psi^{\pi}\left(y_{1}\right), \psi^{\pi}\left(y_{2}\right)\right]=0, \\
{\left[\psi^{\pi}\left(y_{1}\right), \psi^{\pi *}\left(y_{2}\right)\right] } & =\mathrm{i}\left(y_{1} \mid \omega y_{2}\right) 1, \quad y_{1}, y_{2} \in \mathcal{Y} .
\end{aligned}
$$

Note that to any charged CCR representation (3.7) we can associate a regular neutral $\mathrm{CCR}$ representation over $\mathcal{Y}$ equipped with $\operatorname{Re}(\cdot \mid \omega \cdot)$

$$
\mathcal{Y} \ni y \mapsto \mathrm{e}^{\mathrm{i} \phi^{\pi}(y)} \in U(\mathcal{H}),
$$

as well as a $U(1)$ symmetry of charge 1

$$
U(1) \ni \theta \mapsto \mathrm{e}^{\mathrm{i} \theta} \mathbb{1} \in S p(\mathcal{Y}) .
$$

Conversely, charged CCR representations arise when we have a (neutral) CCR representation and the underlying symplectic space is equipped with a charge 1 symmetry. Let us make this precise. Suppose that $(\mathcal{Y}, \omega)$ is a symplectic space and

$$
\mathcal{Y} \ni y \mapsto \mathrm{e}^{\mathrm{i} \phi^{\pi}(y)} \in U(\mathcal{H})
$$

a neutral CCR representation. Suppose that $\mathrm{j}_{\mathrm{ch}}$ is a pseudo-Kähler anti-involution, so that $U(1) \ni \theta \mapsto u_{\theta}=\cos \theta \mathbb{1}+\sin \theta \mathrm{j}_{\mathrm{ch}} \in S p(\mathcal{Y})$ is a charge 1 symmetry. Following Subsection 2.9. we introduce the holomorphic subspace for $\mathrm{j}_{\mathrm{ch}}$, that is

$$
\mathcal{Z}_{\mathrm{ch}}:=\left\{y-\mathrm{ij}_{\mathrm{ch}} y: y \in \mathcal{Y}\right\} \subset \mathbb{C} \mathcal{Y} .
$$

We have a natural identification of the space $\mathcal{Z}_{\text {ch }}$ with $\mathcal{Y}$ :

$$
\mathcal{Y} \ni y \mapsto z=\frac{1}{\sqrt{2}}\left(1-\mathrm{ij}_{\mathrm{ch}}\right) y \text {. }
$$

We use this identification to define charged fields parametrized by $\mathcal{Y}$ :

$$
\psi^{\pi *}(y):=\phi^{\pi}(z), \quad \psi^{\pi}(y):=\phi^{\pi}(\bar{z}) .
$$

Thus we obtain a charged CCR representation over $\mathcal{Y}^{\mathbb{C}}$ with the complex structure given by $\mathrm{j}_{\mathrm{ch}}$ and the antihermitian form

$$
\left(y_{1} \mid \omega y_{2}\right):=y_{1} \omega y_{2}-\mathrm{i} y_{1} \omega \mathrm{j}_{\operatorname{ch}} y_{2} .
$$

3.4. CAR representations. Let $(\mathcal{Y}, \nu)$ be a euclidean space, that is, a real vector space $\mathcal{Y}$ equipped with a positive symmetric form $\nu$.

In this subsection we introduce the concept of a representation of canonical anticommutation relations. The definition that we use is very similar to the well-known definition of a representation of Clifford relations. In the case of CAR representations we assume in addition that the operators satisfying the Clifford relations act on a Hilbert space and are selfadjoint.

CAR representations appear in quantum physics in at least two contexts. First, they describe many body fermionic systems. Second, they describe spinors, that is, representations of Spin groups. In most applications the second meaning is restricted to the finite dimensional case.

Recall that $[A, B]_{+}:=A B+B A$ is the anticommutator of $A$ and $B$. 
DEFINITION 3.8. A representation of the canonical anticommutation relations or a $C A R$ representation over $\mathcal{Y}$ in $\mathcal{H}$ is a linear map

$$
\mathcal{Y} \ni y \mapsto \phi^{\pi}(y) \in B_{\mathrm{h}}(\mathcal{H})
$$

satisfying

$$
\left[\phi^{\pi}\left(y_{1}\right), \phi^{\pi}\left(y_{2}\right)\right]_{+}=2 y_{1} \nu y_{2} \mathbb{1}, \quad y_{1}, y_{2} \in \mathcal{Y} .
$$

The operators $\phi^{\pi}(y)$ are called (fermionic) field operators.

REMARK 3.9. Unfortunately, the analogy between the CAR $(3.9)$ and the CCR $(3.2)$ is somewhat spoiled by the number 2 on the right hand side of (3.9). The reason for this convention is to have the identity $\phi^{\pi}(y)^{2}=y \nu y 1$.

In what follows we assume that we are given a CAR representation (3.8). The operators $\phi^{\pi}(y)$ are called (fermionic) field operators. By complex linearity we can extend the definition of field operators to $w=y_{1}+\mathrm{i} y_{2} \in \mathbb{C} \mathcal{Y}$, where $y_{1}, y_{2} \in \mathcal{Y}$ :

$$
\phi^{\pi}(w):=\phi^{\pi}\left(y_{1}\right)+\mathrm{i} \phi^{\pi}\left(y_{2}\right) .
$$

Definition 3.10. The operators $\phi^{\pi}(w)$ for $w \in \mathbb{C} \mathcal{Y}$ are called complex field operators.

3.5. Charged CAR representations. The concept of CAR relations, as defined in Def. 3.9, is used mainly to describe neutral fermions. Therefore, sometimes we will call them neutral CAR representations. In the context of charged fermions one uses another formalism described in the following definition.

Suppose that $(\mathcal{Y},(\cdot \mid \cdot))$ is a unitary space.

DeFinition 3.11. We say that a linear map

$$
\mathcal{Y} \ni y \mapsto \psi^{\pi}(y) \in B(\mathcal{H})
$$

is a charged CAR representation iff

$$
\begin{aligned}
{\left[\psi^{\pi *}\left(y_{1}\right), \psi^{\pi *}\left(y_{2}\right)\right]_{+} } & =\left[\psi^{\pi}\left(y_{1}\right), \psi^{\pi}\left(y_{2}\right)\right]_{+}=0, \\
{\left[\psi^{\pi}\left(y_{1}\right), \psi^{\pi *}\left(y_{2}\right)\right]_{+} } & =\left(y_{1} \mid y_{2}\right) \mathbb{1}, \quad y_{1}, y_{2} \in \mathcal{Y} .
\end{aligned}
$$

Suppose that $y \mapsto \psi^{\pi}(y)$ is a charged CAR representation. Set

$$
\begin{gathered}
\phi^{\pi}(y):=\psi^{\pi}(y)+\psi^{\pi *}(y), \\
y_{1} \nu y_{2}:=\operatorname{Re}\left(y_{1} \mid y_{2}\right) .
\end{gathered}
$$

Then $\mathcal{Y} \ni y \mapsto \phi^{\pi}(y) \in B_{\mathrm{h}}(\mathcal{H})$ is a neutral CAR representation over the euclidean space $(\mathcal{Y}, \nu)$. In addition, $\mathcal{Y}$ is equipped with a charge 1 symmetry $U(1) \ni \theta \mapsto \mathrm{e}^{\mathrm{i} \theta} \mathbb{1} \in O\left(\mathcal{Y}_{\mathbb{R}}\right)$.

Conversely, charged CAR representations arise when we have a (neutral) CAR representation and the underlying euclidean space is equipped with a $U(1)$ symmetry of charge 1 . Let us make this precise. Suppose that $(\mathcal{Y}, \nu)$ is a euclidean space and

$$
\mathcal{Y} \ni y \mapsto \phi^{\pi}(y) \in B_{\mathrm{h}}(\mathcal{H})
$$

is a neutral CAR representation. Suppose that $\mathrm{j}_{\mathrm{ch}}$ is a Kähler anti-involution, so that $U(1) \ni \theta \mapsto u_{\theta}=\cos \theta \mathbb{1}+\sin \theta \mathrm{j}_{\mathrm{ch}} \in O(\mathcal{Y})$ is a charge 1 symmetry. Following Subsect. 2.9. 
we introduce the holomorphic subspace for $\mathrm{j}_{\mathrm{ch}}$, that is

$$
\mathcal{Z}_{\mathrm{ch}}:=\left\{y-\mathrm{ij}_{\mathrm{ch}} y: y \in \mathcal{Y}\right\} \subset \mathbb{C} \mathcal{Y} .
$$

We have a natural identification of the space $\mathcal{Z}_{\text {ch }}$ with $\mathcal{Y}$ :

$$
\mathcal{Y} \ni y \mapsto z=\frac{1}{2}\left(11-\mathrm{ij}_{\mathrm{ch}}\right) y .
$$

We use this identification to define charged fields as

$$
\psi^{\pi *}(y):=\phi^{\pi}(z), \quad \psi^{\pi}(y):=\phi^{\pi}(\bar{z}) .
$$

Thus we obtain a charged CAR representation over $\mathcal{Y}^{\mathbb{C}}$ with the complex structure given by $\mathrm{j}_{\mathrm{ch}}$ and the scalar product

$$
\left(y_{1} \mid y_{2}\right):=y_{1} \nu y_{2}-\mathrm{i} y_{1} \nu \mathrm{j}_{\mathrm{ch}} y_{2}, \quad y_{1}, y_{2} \in \mathcal{Y} .
$$

4. Fock spaces. In this section we fix our terminology related to bosonic and fermionic Fock spaces. In particular, we introduce the so-called Fock CCR and CAR representations. Unfortunately, no uniform notation concerning this material seems to exist in the literature.

Let us mention, for example, the following works which discuss constructions related to Fock spaces: [BR, De, DG1, GJ, RS2].

4.1. Tensor algebra. Let $\mathcal{Z}$ be a Hilbert space space. Let $\otimes^{n} \mathcal{Z}$ denote the $n$th tensor power of $\mathcal{Z}$. We set $\otimes^{0} \mathcal{Z}:=\mathbb{C}$. The complete tensor algebra over $\mathcal{Z}$ is defined as

$$
\otimes \mathcal{Z}:=\bigoplus_{n=0}^{\infty} \otimes^{n} \mathcal{Z}
$$

It is also sometimes called the full Fock space.

The element $1 \in \otimes^{0} \mathcal{Z}$ is called the vacuum and will be denoted by $\Omega$.

4.2. Operators $\mathrm{d} \Gamma$ and $\Gamma$ on the tensor algebra. Let $\mathcal{Z}, \mathcal{Z}_{1}, \mathcal{Z}_{2}$ be Hilbert spaces. Suppose that $p \in B\left(\mathcal{Z}_{1}, \mathcal{Z}_{2}\right)$ is a contraction. We define

$$
\Gamma^{n}(p):=p^{\otimes n} \in B\left(\otimes^{n} \mathcal{Z}_{1}, \otimes^{n} \mathcal{Z}_{2}\right), \quad \Gamma(p):=\underset{n=0}{\infty} \Gamma^{n}(p) \in B\left(\otimes \mathcal{Z}_{1}, \otimes \mathcal{Z}_{2}\right) .
$$

Likewise, if $h \in C l(\mathcal{Z})$ then we define

$$
\begin{aligned}
\mathrm{d} \Gamma^{n}(h) & :=\sum_{j=1}^{n} \mathbb{1}_{\mathcal{Z}}^{\otimes j-1} \otimes h \otimes \mathbb{1}_{\mathcal{Z}}^{\otimes(n-j)} \in C l\left(\otimes^{n} \mathcal{Z}\right), \\
\mathrm{d} \Gamma(h) & :=\bigoplus_{n=0}^{\infty} \mathrm{d} \Gamma^{n}(h) \in C l(\otimes \mathcal{Z}) .
\end{aligned}
$$

The number operator and the parity operator are defined respectively as

$$
\begin{aligned}
N & :=\mathrm{d} \Gamma(\mathbb{1}), \\
I & :=(-1)^{N}=\Gamma(-\mathbb{1}) .
\end{aligned}
$$

Proposition 4.1. Let $h, h_{1}, h_{2} \in B(\mathcal{Z}), p_{1} \in B\left(\mathcal{Z}, \mathcal{Z}_{1}\right), p_{2} \in B\left(\mathcal{Z}_{1}, \mathcal{Z}_{2}\right),\left\|p_{1}\right\|,\left\|p_{2}\right\| \leq 1$. We then have

$$
\Gamma\left(\mathrm{e}^{h}\right)=\mathrm{e}^{\mathrm{d} \Gamma(h)}, \quad \Gamma\left(p_{2}\right) \Gamma\left(p_{1}\right)=\Gamma\left(p_{2} p_{1}\right), \quad\left[\mathrm{d} \Gamma\left(h_{1}\right), \mathrm{d} \Gamma\left(h_{2}\right)\right]=\mathrm{d} \Gamma\left(\left[h_{1}, h_{2}\right]\right) .
$$


4.3. Bosonic and fermionic Fock spaces. Let $\mathcal{Z}$ be a Hilbert space. If $\sigma \in S_{n}$, then there exists a unique

$$
\Theta(\sigma) \in U\left(\otimes^{n} \mathcal{Z}\right)
$$

such that

$$
\Theta(\sigma) y_{1} \otimes \cdots \otimes y_{n}=y_{\sigma^{-1}(1)} \otimes \cdots \otimes y_{\sigma^{-1}(n)}
$$

Clearly,

$$
S_{n} \ni \sigma \mapsto \Theta(\sigma) \in U\left(\otimes^{n} \mathcal{Z}\right)
$$

is a representation of the permutation group. We define the following operators on $\otimes^{n} \mathcal{Z}$ :

$$
\Theta_{\mathrm{s}}^{n}:=\frac{1}{n !} \sum_{\sigma \in S_{n}} \Theta(\sigma), \quad \Theta_{\mathrm{a}}^{n}:=\frac{1}{n !} \sum_{\sigma \in S_{n}} \operatorname{sgn} \sigma \Theta(\sigma) .
$$

It is easy to check that $\Theta_{\mathrm{s}}^{n}$ and $\Theta_{\mathrm{a}}^{n}$ are orthogonal projections.

We will write $\mathrm{s} / \mathrm{a}$ as a subscript which can mean either s or a. We set

$$
\Gamma_{\mathrm{s} / \mathrm{a}}^{n}(\mathcal{Z}):=\Theta_{\mathrm{s} / \mathrm{a}}^{n} \otimes^{n} \mathcal{Z}, \quad \Gamma_{\mathrm{s} / \mathrm{a}}(\mathcal{Z}):=\oplus_{n=0}^{\infty} \Gamma_{\mathrm{s} / \mathrm{a}}^{n}(\mathcal{Z})=\Theta_{\mathrm{s} / \mathrm{a}} \stackrel{\text { al }}{\otimes} \mathcal{Z} .
$$

$\Gamma_{\mathrm{s} / \mathrm{a}}(\mathcal{Z})$ is called the bosonic, resp. fermionic Fock space [Fo].

Occasionally, we will need the finite particle bosonic, resp. fermionic Fock spaces, denoted $\Gamma_{\mathrm{s} / \mathrm{a}}^{\mathrm{fin}}(\mathcal{Z})$, which are the subspaces of $\Gamma_{\mathrm{s} / \mathrm{a}}(\mathcal{Z})$ consisting of finite sums of $n$-particle vectors.

4.4. $\mathrm{d} \Gamma$ and $\Gamma$ operators on Fock spaces. If $p \in B(\mathcal{Z}, \mathcal{W})$ is a contraction, then $\Gamma^{n}(p)$ maps $\Gamma_{\mathrm{s} / \mathrm{a}}^{n}(\mathcal{Z})$ into $\Gamma_{\mathrm{s} / \mathrm{a}}^{n}(\mathcal{W})$. Hence $\Gamma(p)$ maps $\Gamma_{\mathrm{s} / \mathrm{a}}(\mathcal{Z})$ into $\Gamma_{\mathrm{s} / \mathrm{a}}(\mathcal{W})$. We will use the same symbols $\Gamma^{n}(p)$ and $\Gamma(p)$ to denote the corresponding restricted operators. $\Gamma(p)$ is sometimes called the second quantization of $p$.

If $h \in C l(\mathcal{Z})$, then $\mathrm{d} \Gamma^{n}(h)$ maps $\Gamma_{\mathrm{s} / \mathrm{a}}^{n}(\mathcal{Z})$ into itself. Hence, $\mathrm{d} \Gamma(h) \operatorname{maps} \Gamma_{\mathrm{s} / \mathrm{a}}(\mathcal{Z})$ into itself. We will use the same symbols $\mathrm{d} \Gamma^{n}(h)$ and $\mathrm{d} \Gamma(h)$ to denote the corresponding restricted operators. Perhaps, the correct name of $\mathrm{d} \Gamma(h)$ should be the infinitesimal second quantization of $h$.

4.5. Creation and annihilation operators. Let $\mathcal{Z}$ be a Hilbert space and $z \in \mathcal{Z}$. We consider the bosonic or fermionic Fock space $\Gamma_{\mathrm{s} / \mathrm{a}}(\mathcal{Z})$.

Let $z \in \mathcal{Z}$. We will now define two operators with the domain $\Gamma_{\mathrm{s} / \mathrm{a}}^{\mathrm{fin}}(\mathcal{Z})$.

The creation operator of $z$ is defined as

$$
c(z) \Psi:=\sqrt{n+1} \Theta_{\mathrm{s} / \mathrm{a}}^{n+1} z \otimes \Psi, \quad \Psi \in \Gamma_{\mathrm{s} / \mathrm{a}}^{n}(\mathcal{Z}) .
$$

The annihilation operator of $z$, satisfies

$$
a(z) \Psi:=\sqrt{n}\left(z \mid \otimes \mathbb{1} \Psi, \quad \Psi \in \Gamma_{\mathrm{s} / \mathrm{a}}^{n}(\mathcal{Z}) .\right.
$$

Proposition 4.2.

(1) In the bosonic case, the operators $c(z)$ and $a(z)$ are densely defined and closable. We denote their closures by the same symbols. They satisfy $a(z)^{*}=c(z)$. Therefore we will write $a^{*}(z)$ instead of $c(z)$. We have

$$
\left[a^{*}\left(z_{1}\right), a^{*}\left(z_{2}\right)\right]=0, \quad\left[a\left(z_{1}\right), a\left(z_{2}\right)\right]=0, \quad\left[a\left(z_{1}\right), a^{*}\left(z_{2}\right)\right]=\left(z_{1} \mid z_{2}\right) \mathbb{1} .
$$


(2) In the fermionic case, the operators $c(z)$ and $a(z)$ are densely defined and bounded. $W e$ denote by the same symbols their closures. They satisfy $a(z)^{*}=c(z)$. Therefore we will write $a^{*}(z)$ instead of $c(z)$. We have

$$
\left[a^{*}\left(z_{1}\right), a^{*}\left(z_{2}\right)\right]_{+}=0, \quad\left[a\left(z_{1}\right), a\left(z_{2}\right)\right]_{+}=0, \quad\left[a\left(z_{1}\right), a^{*}\left(z_{2}\right)\right]_{+}=\left(z_{1} \mid z_{2}\right) \mathbb{1} .
$$

4.6. Fock CCR representation. For a Hilbert space $\mathcal{Z}$, we introduce the space

$$
\mathcal{Y}=\operatorname{Re}(\mathcal{Z} \oplus \overline{\mathcal{Z}}):=\{(z, \bar{z}): z \in \mathcal{Z}\}
$$

which will serve as the phase space of our system. It will be equipped with a symplectic form $\omega$ and a Kähler anti-involution j:

$$
\begin{aligned}
(z, \bar{z}) \omega(w, \bar{w}) & :=2 \operatorname{Im}(z \mid w), \\
\mathrm{j}(z, \bar{z}) & :=(\mathrm{i} z, \overline{\mathrm{i} z}) .
\end{aligned}
$$

Proposition 4.3.

$$
\mathcal{Y} \ni y \mapsto W(y)=\mathrm{e}^{\mathrm{i} a^{*}(z)+\mathrm{i} a(z)} \in U\left(\Gamma_{\mathrm{s}}(\mathcal{Z})\right), y=(z, \bar{z}) .
$$

is a regular CCR representation over $(\mathcal{Y}, \omega)$ on $\Gamma_{\mathrm{s}}(\mathcal{Z})$.

Definition 4.4. We call 4.17) the Fock CCR representation.

4.7. Fock CAR representation. Let $\mathcal{Z}, \mathcal{Y}$ and $\mathrm{j}$ remain as in previous subsection. We equip $\mathcal{Y}$ with the structure of a euclidean space with the scalar product $\nu$ :

$$
(z, \bar{z}) \nu(w, \bar{w}):=\operatorname{Re}(z \mid w),
$$

Clearly, $\mathrm{j}$ is a Kähler anti-involution for $\nu$.

Proposition 4.5.

$$
\mathcal{Y} \ni y \mapsto \phi(y)=a^{*}(z)+a(z) \in B_{\mathrm{h}}\left(\Gamma_{\mathrm{s}}(\mathcal{Z})\right), y=(z, \bar{z}) .
$$

is a $C A R$ representation over $(\mathcal{Y}, \nu)$ on $\Gamma_{\mathrm{a}}(\mathcal{Z})$.

Definition 4.6. We call 4.18 the Fock CAR representation.

5. CCR and CAR algebras. In some approaches to quantum physics a considerable importance is attached to the choice of a $*$-algebra, usually a $C^{*}$ - or $W^{*}$-algebra, which is supposed to describe observables of a system [BR, Em, Ha. By choosing a state (or a family of states) and making the corresponding GNS construction, we obtain a representation of this *-algebra in a Hilbert space. This philosophy allows us to study some aspects of quantum systems in a representation independent fashion.

Many authors try to apply this to bosonic and fermionic systems. This is especially natural in the case of fermionic systems, where there exists an obvious choice of a $C^{*}$ algebra describing the CAR over a given euclidean space.

In the bosonic case the situation is more problematic. In particular, for a given symplectic space several natural choices of CCR algebras are possible. We will describe some of them. 
5.1. Weyl CCR algebras. Let $(\mathcal{Y}, \omega)$ be a symplectic space, not necessarily of finite dimension. In this section we introduce the notion of the Weyl CCR $C^{*}$-algebra over $\mathcal{Y}$. It is the $C^{*}$-algebra generated by elements satisfying the Weyl CCR relations over $\mathcal{Y}$. Many mathematical physicists use it in their description of bosonic systems.

Let us start with the definition of the algebraic Weyl CCR algebra over $\mathcal{Y}$.

Definition 5.1. $\mathrm{CCR}_{\mathrm{alg}}^{\mathrm{Weyl}}(\mathcal{Y})$ is defined as the *-algebra generated by elements $W(y)$, $y \in \mathcal{Y}$, satisfying the relations

$$
\begin{aligned}
W\left(y_{1}\right) W\left(y_{2}\right) & =\mathrm{e}^{-\frac{\mathrm{i}}{2} y_{1} \omega y_{2}} W\left(y_{1}+y_{2}\right), \quad y_{1}, y_{2} \in \mathcal{Y} \\
W(y)^{*} & =W(-y), \quad y \in \mathcal{Y} .
\end{aligned}
$$

The following theorem comes from [Sla], see also $[\mathrm{BR}]$ :

THEOREM 5.2. There exist faithful *-representations of $\operatorname{CCR}_{\text {alg }}^{\mathrm{Weyl}}(\mathcal{Y})$. Let $\pi$ be such a representation and let $A \in \mathrm{CCR}_{\mathrm{alg}}^{\mathrm{Weyl}}(\mathcal{Y})$. Then $\|\pi(A)\|$ does not depend on $\pi$.

Thus $\mathrm{CCR}_{\mathrm{alg}}^{\mathrm{Weyl}}(\mathcal{Y})$ possesses a unique $C^{*}$-norm.

Definition 5.3. The Weyl $C C R C^{*}$-algebra is defined as

$$
\operatorname{CCR}^{\text {Weyl }}(\mathcal{Y}):=\left(\operatorname{CCR}_{\text {alg }}^{\text {Weyl }}(\mathcal{Y})\right)^{\mathrm{cpl}}
$$

where cpl denotes the completion. Clearly, $\mathrm{CCR}^{\mathrm{Weyl}}(\mathcal{Y})$ is a $C^{*}$-algebra.

The following isomorphisms are sometimes called Bogoliubov automorphisms.

Proposition 5.4. Let $r \in S p(\mathcal{Y})$. Then there exists a unique *-automorphism

$$
\hat{r}: \mathrm{CCR}^{\mathrm{Weyl}}(\mathcal{Y}) \rightarrow \mathrm{CCR}^{\mathrm{Weyl}}(\mathcal{Y})
$$

such that $\hat{r}(W(y))=W(r y), y \in \mathcal{Y}$.

The following proposition explains the relationship between CCR representations and CCR algebras.

Proposition 5.5. Let $\mathcal{Y} \ni y \mapsto W^{\pi}(y) \in U(\mathcal{H})$ be a CCR representation. Then there exists a unique *-representation $\pi: \mathrm{CCR}^{\mathrm{Weyl}}(\mathcal{Y}) \rightarrow B(\mathcal{H})$ such that $\pi(W(y))=W^{\pi}(y)$. Moreover, $\pi$ is isometric.

5.2. Stone--von Neumann CCR algebras. One can argue that the Weyl CCR algebra is somewhat artificial. In particular, it is a noncommutative analogue of the space of almost periodic functions, which is quite a pathological object. One can therefore try to look for alternatives for Weyl CCR algebras. In this and the next subsection we discuss some alternative algebras describing CCR.

In this subsection we always assume that $(\mathcal{Y}, \omega)$ is a finite dimensional symplectic space. Let us recall the Stone-von Neumann Theorem about the uniqueness of regular CCR relations (see e.g. [BR]).

THEOREM 5.6. Suppose that, for $i=1,2, \mathcal{H}_{i}$ are Hilbert spaces and

$$
\mathcal{Y} \ni y \mapsto W_{i}(y) \in U\left(\mathcal{H}_{i}\right)
$$


are regular $C C R$ representations such that $\operatorname{Span}\left\{W_{i}(y): y \in \mathcal{Y}\right\}$ is weakly dense in $B\left(\mathcal{H}_{i}\right)$. Then there exists $U \in U\left(\mathcal{H}_{1}, \mathcal{H}_{2}\right)$, unique up to a phase factor, such that $W_{2}(y)=$ $U W_{1}(y) U^{*}, y \in \mathcal{Y}$.

Theorem 5.6 suggests the following definition:

Definition 5.7. A Stone-von Neumann CCR algebra over $\mathcal{Y}$ is defined as the von Neumann algebra $B(\mathcal{H})$ for a certain Hilbert space $\mathcal{H}$ with distinguished unitary elements $W(y), y \in \mathcal{Y}$, such that $\mathcal{Y} \ni y \mapsto W(y)$ is a regular CCR representation and $\operatorname{Span}\{W(y): y \in \mathcal{Y}\}$ is weakly dense in $B(\mathcal{H})$. It is denoted $\operatorname{CCR}^{\mathrm{SvN}}(\mathcal{Y})$.

By Theorem 5.6. $\mathrm{CCR}^{\mathrm{SvN}}(\mathcal{Y})$ is defined uniquely up to a unitary equivalence. Clearly, $\mathrm{CCR}^{\mathrm{SvN}}(\mathcal{Y})$ is not very interesting as a von Neumann-algebra - it is isomorphic to the usual type I factor. What is interesting is the category of Bogoliubov automorphisms between these algebras, described in the following proposition:

Proposition 5.8.

(1) Let $r \in S p(\mathcal{Y})$. Then there exists a unique spatially implementable $*$-automorphism $\hat{r}$ of $\mathrm{CCR}^{\mathrm{SvN}}(\mathcal{Y})$ such that $\hat{r}(W(y))=W(r y), y \in \mathcal{Y}$.

(2) Let $\mathcal{Y}_{1}$ be a symplectic subspace of $\mathcal{Y}$. Then there is a unique embedding of $\mathrm{CCR}^{\mathrm{SvN}}\left(\mathcal{Y}_{1}\right)$ in $\mathrm{CCR}^{\mathrm{SvN}}(\mathcal{Y})$, such that, for $y \in \mathcal{Y}_{1}, W(y)$ in the sense of $\mathrm{CCR}^{\mathrm{SvN}}\left(\mathcal{Y}_{1}\right)$ coincide with $W(y)$ in the sense of $\mathrm{CCR}^{\mathrm{SvN}}(\mathcal{Y})$.

5.3. Regular CCR algebras. Let $(\mathcal{Y}, \omega)$ be a symplectic space of arbitrary dimension. By $\operatorname{FinSym}(\mathcal{Y})$ we will denote the set of finite dimensional symplectic subspaces of $\mathcal{Y}$.

In this subsection we introduce the notion of the regular CCR algebra over $\mathcal{Y}$. In the literature, it is rarely used. Weyl CCR algebras are more common. Nevertheless, it is a natural construction, and we will find it useful, especially to describe charged bosonic systems. Its use was advocated by I.Segal.

Let $\mathcal{Y}_{1}, \mathcal{Y}_{2} \in \operatorname{FinSym}(\mathcal{Y})$ and $\mathcal{Y}_{1} \subset \mathcal{Y}_{2}$. We can define their Stone-von Neumann CCR algebras, as in Definition 5.7. By Proposition 5.8, we have a natural embedding

$$
\mathrm{CCR}^{\mathrm{SvN}}\left(\mathcal{Y}_{1}\right) \subset \operatorname{CCR}^{\mathrm{SvN}}\left(\mathcal{Y}_{2}\right) \text {. }
$$

We can define the algebraic regular CCR $*$-algebra as the inductive limit of Stone-von Neumann CCR algebras:

DEFINITION 5.9. We set

$$
\operatorname{CCR}_{\text {alg }}^{\text {reg }}(\mathcal{Y}):=\bigcup_{\mathcal{Y}_{1} \in \operatorname{FinSym}(\mathcal{Y})} \operatorname{CCR}^{\operatorname{SvN}}\left(\mathcal{Y}_{1}\right) .
$$

Clearly, $\mathrm{CCR}_{\text {alg }}^{\mathrm{reg}}(\mathcal{Y})$ is a $*$-algebra equipped with a $C^{*}$-norm.

Definition 5.10. We define the regular CCR $C^{*}$-algebra over $\mathcal{Y}$ as

$$
\operatorname{CCR}^{\mathrm{reg}}(\mathcal{Y}):=\left(\operatorname{CCR}_{\text {alg }}^{\mathrm{reg}}(\mathcal{Y})\right)^{\mathrm{cpl}},
$$

where the completion is with respect to the norm defined above.

Clearly, $\operatorname{CCR}^{\text {reg }}(\mathcal{Y})$ is a generalization of the Stone-von Neumann algebra $\operatorname{CCR}(\mathcal{Y})$ from Definition 5.7 . 
We have an obvious extension of Proposition 5.8 .

Proposition 5.11. Let $r \in S p(\mathcal{Y})$. Then there is a unique $*$-isomorphism $\hat{r}: \mathrm{CCR}^{\mathrm{reg}}(\mathcal{Y})$ $\rightarrow \operatorname{CCR}^{\mathrm{reg}}(\mathcal{Y})$ such that $\hat{r}(W(y))=W(r y), y \in \mathcal{Y}$, and if $\mathcal{Y}_{1} \in \operatorname{FinSym}(\mathcal{Y})$, then $\hat{r}$ restricted to $\mathrm{CCR}^{\mathrm{SvN}}\left(\mathcal{Y}_{1}\right) \rightarrow \mathrm{CCR}^{\mathrm{SvN}}\left(r \mathcal{Y}_{1}\right)$ is $\sigma$-weakly continuous.

Among the Bogoliubov automorphism one can distinguish the parity, that is $\alpha:=\widehat{-1}$. Clearly, $\alpha$ is an involution. Elements of $\mathrm{CCR}^{\text {reg }}(\mathcal{Y})$ fixed by $\alpha$ are called even and form a subalgebra denoted $\operatorname{CCR}_{0}^{\text {reg }}(\mathcal{Y})$.

Proposition 5.12. Let $\mathcal{Y} \ni y \mapsto W^{\pi}(y) \in U(\mathcal{H})$ be a regular CCR representation. Then there exists a unique $*$-representation $\pi: \operatorname{CCR}^{\text {reg }}(\mathcal{Y}) \rightarrow B(\mathcal{H})$ such that $\pi(W(y))=$ $W^{\pi}(y), y \in \mathcal{Y}$, and which is $\sigma$-weakly continuous on the subalgebras $\operatorname{CCR}^{\mathrm{SvN}}\left(\mathcal{Y}_{1}\right) \subset$ $\operatorname{CCR}^{\mathrm{reg}}(\mathcal{Y})$ for $\mathcal{Y}_{1} \in \operatorname{FinSym}(\mathcal{Y})$. Moreover, $\pi$ is isometric.

5.4. $C^{*}$-CAR algebras. In the case of the CAR, there is an obvious well-known choice of a $C^{*}$-algebra, which we recall in this subsection. It is discussed in many places in the literature, see for instance $\mathrm{Ar} 3, \mathrm{PR}$.

Throughout the subsection we assume that $(\mathcal{Y}, \nu)$ is a euclidean space.

Definition 5.13. The complex unital $*$-algebra generated by selfadjoint elements $\phi(y)$ depending linearly on $y \in \mathcal{Y}$ satisfying

$$
\left[\phi\left(y_{1}\right), \phi\left(y_{2}\right)\right]_{+}=2 y_{1} \nu y_{2} \mathbb{1}, \quad y_{1}, y_{2} \in \mathcal{Y},
$$

will be denoted by $\operatorname{CAR}^{\operatorname{alg}}(\mathcal{Y})$.

It is easy to prove the following proposition:

Proposition 5.14. There exists a unique $C^{*}$-norm on $\operatorname{CAR}^{\text {alg }}(\mathcal{Y})$.

DeFinition 5.15. We set

$$
\operatorname{CAR}^{C^{*}}(\mathcal{Y}):=\left(\operatorname{CAR}^{\text {alg }}(\mathcal{Y})\right)^{\text {cpl }},
$$

where the completion is with respect to the $C^{*}$ norm defined above.

REMARK 5.16. In the literature, $\operatorname{CAR}^{C^{*}}(\mathcal{Y})$ is usually denoted $\operatorname{CAR}(\mathcal{Y})$. Our more complicated notation is motivated by the fact that there exist other natural *algebras that describe the CAR over $\mathcal{Y}$. Another choice, discussed in [DG2, $P R]$, is the $W^{*}$-algebra $\mathrm{CAR}^{W^{*}}(\mathcal{Y})$, obtained by taking the weak closure in the GNS representation for the unique tracial state on $\mathrm{CAR}^{C^{*}}(\mathcal{Y})$.

Clearly, $\operatorname{CAR}^{C^{*}}(\mathcal{Y})$ is a $C^{*}$ algebra. It coincides with $\mathrm{CAR}^{C^{*}}\left(\mathcal{Y}^{\mathrm{cpl}}\right)$. Hence it is enough to assume that $\mathcal{Y}$ is a real Hilbert space.

The following proposition describes the so-called fermionic Bogoliubov automorphisms:

Proposition 5.17. If $r \in O(\mathcal{Y})$, there exists a unique $*$-automorphism $\hat{r}$ of $\operatorname{CAR}^{C^{*}}(\mathcal{Y})$ satisfying $\hat{r}(\phi(y))=\phi(r y)$.

Among the Bogoliubov automorphisms a special role is played by the parity, that is $\alpha:=\widehat{-1}$. Clearly, $\alpha$ is an involution. Elements of $\operatorname{CAR}^{C^{*}}(\mathcal{Y})$ fixed by $\alpha$ are called even and form a subalgebra denoted $\operatorname{CAR}_{0}^{C^{*}}(\mathcal{Y})$. 
The relationship between $\mathrm{CAR}$ representations and the algebra $\operatorname{CAR}^{C^{*}}(\mathcal{Y})$ is given by the following proposition:

Proposition 5.18. If $\mathcal{Y} \ni y \mapsto \phi^{\pi}(y) \in B_{\mathrm{h}}(\mathcal{H})$ is a CAR representation, then there exists a unique *-homomorphism of $C^{*}$-algebras

$$
\pi: \operatorname{CAR}^{C^{*}}(\mathcal{Y}) \rightarrow B(\mathcal{H})
$$

such that $\pi(\phi(y))=\phi^{\pi}(y), y \in \mathcal{Y}$.

$\mathrm{CAR}^{C^{*}}(\mathcal{Y})$ belongs to one of the best known classes of $C^{*}$-algebras, as seen from the following proposition:

Proposition 5.19. If $\mathcal{Y}$ is infinite dimensional separable, then $\operatorname{CAR}^{C^{*}}(\mathcal{Y})$ is isomorphic to the so-called uniformly hyperfinite $C^{*}$-algebra of the type $2^{\infty}$, sometimes denoted $U H F\left(2^{\infty}\right)$.

6. Quantization of neutral systems. In this and the next section we describe the positive quantization of classical linear systems. It is natural to divide the discussion into two sections: the first about neutral and the second about charged systems. In both sections we describe bosonic and fermionic systems. Then we consider discrete symmetries: the time reversal and the charge reversal.

In the neutral formalism the classical phase space $\mathcal{Y}$ is real and is equipped with a symplectic form $\omega$ in the bosonic case, resp. with a positive scalar product $\nu$ in the fermionic case. The dynamics describing the time evolution satisfies $r_{t} \in \mathcal{S} p(\mathcal{Y})$, resp. $r_{t} \in O(\mathcal{Y})$. The problem adressed in this section is to find a CCR representation, resp. a CAR representation, on a Hilbert space $\mathcal{H}$ and a positive selfadjoint operator $H$ on $\mathcal{H}$ such that $\mathrm{e}^{\mathrm{i} t H}$ implements $r_{t}$.

We will do it by finding a Kähler anti-involution that commutes with the dynamics, and thus leads to a Fock representation in which the dynamics is implementable.

It turns out that this is easy in the fermionic case. The bosonic case is more technical. In particular, one needs to assume that the dynamics is stable, which roughly means that the classical Hamiltonian is positive.

One often assumes that the dynamics $\left\{r_{t}\right\}_{t \in \mathbb{R}}$ is a part of a larger group of symmetries $G$. In other words, our starting point is a homorphism of a group $G$ into $\mathcal{S} p(\mathcal{Y})$, resp. $O(\mathcal{Y})$. One often asks whether the action of $G$ can be implemented in the Hilbert space $\mathcal{H}$ by unitary or, sometimes, antiunitary operators.

\subsection{Neutral bosonic systems}

6.1.1. Algebraic quantization of a symplectic dynamics. Let $(\mathcal{Y}, \omega)$ be a symplectic space. Let $\mathbb{R} \ni t \mapsto r_{t} \in S p(\mathcal{Y})$ be a 1-parameter group.

It is easy to describe the quantum counterpart of the above classical dynamical system. We take one of the CCR algebras over $(\mathcal{Y}, \omega)$, say $\operatorname{CCR}^{\mathrm{Weyl}}(\mathcal{Y})$, and equip it with the group of Bogoliubov automorphisms $\hat{r}_{t}$, defined by

$$
\hat{r}_{t}(W(y))=W\left(r_{t} y\right), \quad y \in \mathcal{Y} .
$$


6.1.2. Stable symplectic dynamics. Typical symplectic dynamics that appear in physics have positive Hamiltonians. We will call such dynamics stable. We will see that (under some technical conditions) such dynamics lead to uniquely defined Fock representations.

It is easy to make the concept of stability precise if $\operatorname{dim} \mathcal{Y}<\infty$. In this case $\mathcal{Y}$ has a natural topology. We, of course, assume that the dynamics $t \mapsto r_{t}$ is continuous. Let $a$ be its generator, so that $r_{t}=\mathrm{e}^{t a}$. Clearly, the form $\beta$ defined by

$$
y_{1} \beta y_{2}:=y_{1} \omega a y_{2}, \quad y_{1}, y_{2} \in \mathcal{Y},
$$

is symmetric. We say that the group $t \mapsto r_{t}$ is stable if $\beta$ is strictly positive.

6.1.3. Kähler structure for a weakly stable symplectic dynamics. To generalize the concept of stability to infinite dimensions, we need to equip $(\mathcal{Y}, \omega)$ with a topology. There are various possibilities to do this, let us consider the simplest one.

Definition 6.1. We say that $\left(\mathcal{Y}, \omega, \beta,\left(r_{t}\right)_{t \in \mathbb{R}}\right)$ is a weakly stable dynamics if the following conditions are true:

(1) $\beta$ is a positive definite symmetric form. We equip $\mathcal{Y}$ with the norm $\|y\|_{\text {en }}:=(y \cdot \beta y)^{\frac{1}{2}}$. We denote by $\mathcal{Y}_{\text {en }}$ the completion of $\mathcal{Y}$ with respect to this norm.

(2) $t \mapsto r_{t} \in S p(\mathcal{Y})$ is bounded and strongly continuous. Thus we can extend $r_{t}$ to a strongly continuous group on $\mathcal{Y}_{\text {en }}$ and define its generator $a$, so that $r_{t}=\mathrm{e}^{t a}$.

(3) $\operatorname{Ker} a=\{0\}$, or equivalently, $\bigcap_{t \in \mathbb{R}} \operatorname{Ker}\left(r_{t}-\mathbb{1}\right)=\{0\}$.

(4) $\mathcal{Y} \subset$ Dom $a$ and

$$
y_{1} \beta y_{2}=y_{1} \omega a y_{2}, \quad y_{1}, y_{2} \in \mathcal{Y} .
$$

If in addition $\omega$ is bounded for the topology given by $\beta$, so that it can be extended to the whole $\mathcal{Y}_{\text {en }}$, we will say that the dynamics is strongly stable. In this case $\left(\mathcal{Y}_{\text {en }}, \omega\right)$ is a symplectic space.

Note that $\beta$ has two roles: it endows $\mathcal{Y}$ with a topology and it is the Hamiltonian for $r_{t}$.

TheOrem 6.2. Let $\left(\mathcal{Y}, \omega, \beta,\left(r_{t}\right)_{t \in \mathbb{R}}\right)$ be a weakly stable dynamics. Then

(1) $r_{t}$ are orthogonal transformations on the real Hilbert space $\mathcal{Y}_{\mathrm{en}}$.

(2) $a$ is antiselfadjoint and Ker $a=\{0\}$.

(3) Let $h:=\sqrt{a a^{*}}=\sqrt{a^{*} a}$. The polar decomposition

$$
a=: h \mathrm{j}=\mathrm{j} h
$$

defines an anti-involution $\mathrm{j}$ on $\mathcal{Y}_{\mathrm{en}}$.

(4) $h$ is a positive selfadjoint operator.

(5) The dynamics is strongly stable iff $h \geq C$ for some $C>0$.

Recall that given a strictly positive operator $h$ on $\mathcal{Y}_{\text {en }}$ we can define a scale of Hilbert spaces $h^{s} \mathcal{Y}_{\text {en }}$. Then $r_{t}$ and $\mathrm{j}$ are bounded on $\mathcal{Y}_{\text {en }} \cap h^{s} \mathcal{Y}_{\text {en }}$ for the norm of $h^{s} \mathcal{Y}_{\text {en }}$. Let $r_{s, t}$ and $\mathrm{j}_{s}$ denote their extensions. Similarly, $a$ and $h$ are closable on $\mathcal{Y}_{\text {en }} \cap h^{s} \mathcal{Y}_{\text {en }}$ for the norm $h^{s} \mathcal{Y}_{\text {en }}$. Let $a_{s}, h_{s}$ denote their closures. Clearly, for any $s, a_{s}=h_{s} \mathrm{j}_{s}=\mathrm{j}_{s} h_{s}$ is the polar decomposition, $\mathrm{j}_{s}$ is an orthogonal anti-involution and $r_{s, t}=\mathrm{e}^{t a_{s}}$ is an orthogonal 1-parameter group. 
Let $\cdot s$ denote the natural scalar product on $h^{s} \mathcal{Y}_{\text {en }}$. Let us express the scalar product and the symplectic form in terms of $(\cdot \mid \beta \cdot)$ :

$$
y_{1} \cdot y_{2}=y_{1} \beta h^{-2 s} y_{2}=\left(h^{-2 s} y_{1}\right) \beta y_{2}, \quad y_{1} \omega y_{2}=y_{1} \beta a^{-1} y_{2}=\left(a^{-1} y_{1}\right) \beta y_{2} .
$$

Note that the symplectic form does not need to be everywhere defined.

Of particular interest for us is the case $s=\frac{1}{2}$, for which we introduce the notation $\mathcal{Y}_{\text {dyn }}:=h^{\frac{1}{2}} \mathcal{Y}_{\text {en }}$. In what follows we drop the subscript $s=\frac{1}{2}$ from $r_{s, t}, \mathrm{j}_{s}, \cdot_{s}, a_{s}$ and $h_{s}$.

Proposition 6.3. $\mathcal{Y}_{\text {dyn }}$ equipped with $(\cdot, \omega, \mathrm{j})$ is a Kähler space.

Clearly, $h$ is positive and we have a dynamics on $\mathcal{Y}_{\text {dyn }}$

$$
r_{t}=\mathrm{e}^{\mathrm{j} t h} .
$$

6.1.4. Fock quantization of symplectic dynamics. In this subsection we drop the subscript dyn from $\mathcal{Y}_{\text {dyn }}$. Let $\mathcal{Z}$ be the holomorphic subspace of $\mathbb{C} \mathcal{Y}$ for the Kähler antiinvolution $\mathrm{j}$ constructed in Thm. 6.2 .

Clearly $h$ commutes with $\mathrm{j}$, hence its complexification $h_{\mathbb{C}}$ preserves $\mathcal{Z}$. We set $h_{\mathcal{Z}}:=$ $\left.h_{\mathbb{C}}\right|_{\mathcal{Z}}$, which is a positive selfadjoint operator on $\mathcal{Z}$ with $\operatorname{Ker} h_{\mathcal{Z}}=\{0\}$.

Likewise $\left(r_{t}\right)_{\mathbb{C}}$ preserves $\mathcal{Z}$ and we have

$$
\left.\left(r_{t}\right)_{\mathbb{C}}\right|_{\mathcal{Z}}=\mathrm{e}^{\mathrm{i} t h_{\mathcal{Z}}}
$$

For $y \in \mathcal{Y}$ define the field operators

$$
\phi(y):=a^{*}\left(\frac{1-\mathrm{ij}}{2} y\right)+a\left(\frac{1-\mathrm{ij}}{2} y\right) .
$$

Then

$$
\mathcal{Y} \ni y \mapsto \mathrm{e}^{\mathrm{i} \phi(y)} \in U\left(\Gamma_{\mathrm{s}}(\mathcal{Z})\right)
$$

is a Fock CCR representation. Introduce the positive operator $H:=\mathrm{d} \Gamma\left(h_{\mathcal{Z}}\right)$ on $\Gamma_{\mathrm{s}}(\mathcal{Z})$. We have

$$
\mathrm{e}^{\mathrm{i} t H} \phi(y) \mathrm{e}^{-\mathrm{i} t H}=\phi\left(r_{t} y\right)
$$

Definition 6.4. 6.3 is called the positive energy Fock quantization for the weakly stable dynamics $t \mapsto r_{t}$.

EXAMPLE 6.5. Let us describe a typical application of the neutral bosonic formalism.

Let $\mathcal{Y}$ be the space of real solutions of the Klein-Gordon equation on the Minkowski space that have a compact support on any space-like hypersurface. It is equipped with a natural symplectic form obtained by integrating the well-known conserved current on any spacelike hypersurface. $\mathbb{C} \mathcal{Y}$ is the space of complex solutions and $\mathcal{Z}$ is the space of positive frequency solutions of the Klein-Gordon equation.

More generally, instead of the Minkowski space we can take a stationary globally hyperbolic space-time and allow for a position-dependent mass. 


\subsection{Neutral fermionic systems}

6.2.1. Algebraic quantization of an orthogonal dynamics. Let $(\mathcal{Y}, \nu)$ be a real Hilbert space. We think of it as the phase space of a fermionic system. A strongly continuous 1-parameter group $\left\{r_{t}\right\}_{t \in \mathbb{R}}$ with $r_{t} \in O(\mathcal{Y})$ will be called an orthogonal dynamics. We view it as a classical dynamical system.

We choose $\operatorname{CAR}^{C^{*}}(\mathcal{Y})$ as the field algebra of our system. It is equipped with the 1-parameter group of Bogoliubov automorphisms $\hat{r}_{t}$, defined by

$$
\hat{r}_{t}(\phi(y))=\phi\left(r_{t} y\right), \quad y \in \mathcal{Y} .
$$

In quantum physics only even fermionic operators are observable. Therefore, it seems natural to use the even subalgebra $\operatorname{CAR}_{0}^{C^{*}}(\mathcal{Y})$ as the observable algebra.

6.2.2. Kähler structure for a nondegenerate orthogonal dynamics. Let a be the generator of $r_{t}$, so that $r_{t}=\mathrm{e}^{t a}$ and $a$ is antiselfadjoint.

Definition 6.6. We say that the dynamics $t \mapsto r_{t} \in O(\mathcal{Y})$ is nondegenerate if

$$
\text { Ker } a=\{0\} \text {, or equivalently } \bigcap_{t \in \mathbb{R}} \operatorname{Ker}\left(r_{t}-\mathbb{1}\right)=\{0\} \text {. }
$$

TheOREM 6.7. Set $h:=\sqrt{a a^{*}}=\sqrt{a^{*} a}$. The polar decomposition

$$
a=: h \mathrm{j}=\mathrm{j} h
$$

defines an anti-involution $\mathrm{j}$ on $\mathcal{Y}$, which is Kähler for $\nu$.

Clearly, $h$ is positive and $r_{t}=\mathrm{e}^{\mathrm{j} t h}$.

6.2.3. Fock quantization of orthogonal dynamics. Let $\mathcal{Z}$ be the holomorphic subspace of $\mathbb{C} \mathcal{Y}$ for the Kähler anti-involution j.

The operator $h_{\mathbb{C}}$ commutes with $\mathrm{j}$. Hence, it preserves $\mathcal{Z}$. We set $h_{\mathcal{Z}}:=\left.h_{\mathbb{C}}\right|_{\mathcal{Z}}$. Note that $h_{\mathcal{Z}}$ is positive.

Consider the Fock representation associated with the Kähler anti-involution j

$$
\mathcal{Y} \ni y \mapsto \phi(y):=a^{*}\left(\frac{1-\mathrm{ij}}{2} y\right)+a\left(\frac{1-\mathrm{ij}}{2} y\right) \in B_{\mathrm{h}}\left(\Gamma_{\mathrm{a}}(\mathcal{Z})\right),
$$

and the positive operator $H:=\mathrm{d} \Gamma\left(h_{\mathcal{Z}}\right)$ on $\Gamma_{\mathrm{a}}(\mathcal{Z})$. We have

$$
\mathrm{e}^{\mathrm{i} t H} \phi(y) \mathrm{e}^{-\mathrm{i} t H}=\phi\left(r_{t} y\right) \text {. }
$$

Definition 6.8. (6.6) is called the positive energy Fock quantization for the dynamics $t \mapsto r_{t}$.

EXAMPLE 6.9. Let us describe a typical application of the neutral fermionic formalism.

Let $\mathcal{Y}$ be the space of solutions of the Dirac equation on the Minkowski space satisfying the Majorana condition that have a compact support on any space-like hypersurface. It is equipped with a natural scalar product obtained by integrating the well-known conserved current on any spacelike hypersurface. $\mathbb{C} \mathcal{Y}$ is the space of all solutions, without imposing the Majorana condition, and $\mathcal{Z}$ is the space of positive frequency solutions of the Dirac equation.

More generally, instead of the Minkowski space we can take a stationary globally hyperbolic space-time and allow for a position-dependent mass. 


\subsection{Time reversal in neutral systems}

6.3.1. Algebraic quantization of time reversal. Let $(\mathcal{Y}, \omega)$ be a symplectic space space with a dynamics $\mathbb{R} \ni t \mapsto r_{t} \in S p(\mathcal{Y})$ in the bosonic case, or let $(\mathcal{Y}, \nu)$ be a real Hilbert space with a dynamics $\mathbb{R} \ni t \mapsto r_{t} \in O(\mathcal{Y})$ in the fermionic case.

Definition 6.10. A map $\tau \in L(\mathcal{Y})$ is called a time reversal if $\tau r_{t}=r_{-t} \tau$ and

- is antisymplectic and $\tau^{2}=11$ in the bosonic case,

- is orthogonal and $\tau^{2}=\mathbb{1}$ or $\tau^{2}=-\mathbb{1}$ in the fermionic case.

In the bosonic case we define an antilinear $*$-homomorphism of the algebra $\operatorname{CCR}^{\text {Weyl }}(\mathcal{Y})$ by setting $\hat{\tau}(W(y)):=W(\tau y)$. Clearly, $\hat{\tau}^{2}$ is the identity.

In the fermionic case, we define an antilinear *-homomorphism of the algebra $\operatorname{CAR}^{C^{*}}(\mathcal{Y})$ by setting $\hat{\tau}(\phi(y)):=\phi(\tau y)$. Clearly, restricted to $\operatorname{CAR}_{0}^{C^{*}}(\mathcal{Y}), \hat{\tau}^{2}$ is the identity.

6.3.2. Fock quantization of time reversal. In the bosonic case we assume that the dynamics is weakly stable, in the fermionic case we assume that it is nondegenerate. In both cases we can introduce $a, \mathrm{j}, h$. Note that we have

$$
\tau a=-a \tau, \quad \tau \mathrm{j}=-\mathrm{j} \tau, \quad \tau h=h \tau .
$$

Recall that $\tau_{\overline{\mathbb{C}}}$ denotes the antilinear extension of $\tau$ to $\mathbb{C} \mathcal{Y}$. Note that $\tau_{\overline{\mathbb{C}}}$ preserves $\mathcal{Z}$. We write $\tau_{\mathcal{Z}}:=\left.\tau_{\overline{\mathbb{C}}}\right|_{\mathcal{Z}}$. Clearly, $\tau_{\mathcal{Z}}$ is antiunitary and

$$
\begin{array}{r}
\tau_{\mathcal{Z}} h_{\mathcal{Z}}=h_{\mathcal{Z}} \tau_{\mathcal{Z}} . \\
\tau_{\mathcal{Z}}^{2}=\mathbb{1} \text { in the bosonic case, } \\
\tau_{\mathcal{Z}}^{2}=\mathbb{1} \text { or } \tau_{\mathcal{Z}}^{2}=-\mathbb{1} \text { in the fermionic case. }
\end{array}
$$

Consider the positive energy quantization of the dynamics on the Fock space $\Gamma_{\mathrm{s} / \mathrm{a}}(\mathcal{Z})$. On the quantum level the time reversal is defined as the antiunitary map $T:=\Gamma\left(\tau_{\mathcal{Z}}\right)$. We have

$$
\begin{gathered}
T H T^{-1}=H, \quad T \mathrm{e}^{\mathrm{i} t H} T^{-1}=\mathrm{e}^{-\mathrm{i} t H}, \\
T \phi(y) T^{-1}=\phi(\tau y), \quad y \in \mathcal{Y} .
\end{gathered}
$$

Note that

$$
\begin{aligned}
T^{2} & =\mathbb{1} \text { in the bosonic case, } \\
T^{2}=\mathbb{1} \text { or } T^{2} & =I \text { in the fermionic case, }
\end{aligned}
$$

where $I$ is the parity operator defined in 4.14).

7. Quantization of charged systems. In the charged formalism, the classical system is described by a complex vector space $\mathcal{Y}$. In the bosonic case, it is equipped with an antihermitian form $(\cdot \mid \omega \cdot)$ - we say that it is a charged symplectic space. The dynamics $\left(r_{t}\right)_{t \in \mathbb{R}}$ describing the time evolution is assumed to preserve $(\cdot \mid \omega \cdot)$, we say that $r_{t}$ is charged symplectic. In the fermionic case it is equipped with a positive scalar product $(\cdot \mid \cdot)$ and without decreasing generality we can assume that it is complete - it is a complex Hilbert space. The dynamics $\left(r_{t}\right)_{t \in \mathbb{R}}$ preserves $(\cdot \mid \cdot)$ - it is unitary. 
By a positive energy quantization of a charged classical system we mean the construction of a charged CCR (resp. CAR) representation $y \mapsto \psi(y)$ on a Hilbert space $\mathcal{H}$ and a positive selfadjoint operator $H$ on $\mathcal{H}$ such that $\mathrm{e}^{\mathrm{i} t H}$ implements $r_{t}$.

The complex structure of $\mathcal{Y}$ is responsible for the action of a $U(1)$ symmetry $\left(\mathrm{e}^{\mathrm{i} \theta}\right)_{\theta \in[0,2 \pi]}$. On the level of the Fock representation it is implemented by the charge operator $Q$.

Charged systems can be viewed as special cases of neutral systems equipped in addition with a certain symmetry. Recall that a homomorphism $U(1) \ni \theta \mapsto u_{\theta} \in L(\mathcal{Y})$ is a $U(1)$ symmetry of charge 1 if there exists an anti-involution $j_{\text {ch }}$ such that $u_{\theta}=$ $\cos \theta \mathbb{1}+\sin \theta \mathrm{j}_{\mathrm{ch}}$. We assume that it preserves the symplectic, resp. euclidean form $\omega$, resp. $\nu$, which is equivalent to saying that $j_{c h}$ is pseudo-Kähler, resp. Kähler. We also assume that the dynamics $r_{t}$ commutes with the symmetry, which is equivalent to saying that $\mathrm{j}_{\mathrm{ch}}$ commutes with $r_{t}$.

If we equip $\mathcal{Y}$ with a complex structure given by $\mathrm{j}_{\mathrm{ch}}$, then the symmetry $u_{\theta}$ becomes just the multiplication by $\mathrm{e}^{\mathrm{i} \theta}$. It is then natural to replace the real bilinear forms $\omega$, resp. $\nu$ by closely related sesquilinear forms $(\cdot \mid \omega \cdot)$, resp. $(\cdot \mid \cdot)$. The invariance of the dynamics with respect to the charge symmetry is now expressed by the fact that the dynamics is complex linear.

At the end of this section, we will discuss the charge reversal and the time reversal for charged systems.

\subsection{Charged bosonic systems}

7.1.1. Algebraic quantization of a charged symplectic dynamics. Let $(\mathcal{Y},(\cdot \mid \omega \cdot))$ be a charged symplectic space. Let $t \mapsto r_{t} \in C h S p(\mathcal{Y})$ be a charged symplectic dynamics.

By taking $\operatorname{Re}\left(y_{1} \mid \omega y_{2}\right)$ we can view $\mathcal{Y}_{\mathbb{R}}$ as a real symplectic space. We choose $\operatorname{CCR}^{\text {reg }}\left(\mathcal{Y}_{\mathbb{R}}\right)$ as the field algebra of our system. This algebra is generated (in the sense described in Subsect. 5.3 by the Weyl elements denoted $\mathrm{e}^{\mathrm{i} \psi(y)+\mathrm{i} \psi^{*}(y)}, y \in \mathcal{Y}$, satisfying the relations

$$
\mathrm{e}^{\mathrm{i} \psi\left(y_{1}\right)+\mathrm{i} \psi^{*}\left(y_{1}\right)} \mathrm{e}^{\mathrm{i} \psi\left(y_{2}\right)+\mathrm{i} \psi^{*}\left(y_{2}\right)}=\mathrm{e}^{-\mathrm{i} \operatorname{Re}\left(y_{1} \mid \omega y_{2}\right)} \mathrm{e}^{\mathrm{i} \psi\left(y_{1}+y_{2}\right)+\mathrm{i} \psi^{*}\left(y_{1}+y_{2}\right)} .
$$

We can equip $\mathrm{CCR}^{\mathrm{reg}}\left(\mathcal{Y}_{\mathbb{R}}\right)$ with the automorphism groups $\widehat{\mathrm{e}^{\mathrm{i} \theta}}$ and $\hat{r}_{t}$ defined by

$$
\widehat{\mathrm{e}^{\mathrm{i} \theta}}\left(\mathrm{e}^{\mathrm{i} \psi(y)+\mathrm{i} \psi^{*}(y)}\right)=\mathrm{e}^{\mathrm{i} \psi\left(\mathrm{e}^{\mathrm{i} \theta} y\right)+\mathrm{i} \psi^{*}\left(\mathrm{e}^{\mathrm{i} \theta} y\right)}, \quad \hat{r}_{t}\left(\mathrm{e}^{\mathrm{i} \psi(y)+\mathrm{i} \psi^{*}(y)}\right)=\mathrm{e}^{\mathrm{i} \psi\left(r_{t} y\right)+\mathrm{i} \psi^{*}\left(r_{t} y\right)} .
$$

As the observable algebra it is natural to choose the so-called gauge invariant regular $C C R$ algebra $\operatorname{CCR}_{\mathrm{gi}}^{\mathrm{reg}}(\mathcal{Y})$, which is defined as the set of elements of $\operatorname{CCR}^{\text {reg }}\left(\mathcal{Y}_{\mathbb{R}}\right)$ fixed by $\widehat{\mathrm{e}^{\mathrm{i} \theta}}$. Note that $\operatorname{CCR}_{\text {gi }}^{\mathrm{reg}}(\mathcal{Y})$ is contained in the even algebra $\operatorname{CCR}_{0}^{\mathrm{reg}}\left(\mathcal{Y}_{\mathbb{R}}\right)$ and is preserved by the dynamics $\hat{r}_{t}$.

REMARK 7.1. In this subsection, for the field algebra of our system we preferred to choose $\operatorname{CCR}^{\text {reg }}\left(\mathcal{Y}_{\mathbb{R}}\right)$ instead of $\mathrm{CCR}^{\text {Weyl }}\left(\mathcal{Y}_{\mathbb{R}}\right)$. This is motivated by the fact that the only element left invariant by the gauge symmetry $\widehat{\mathrm{e}^{\mathrm{i} \theta}}$ in $\operatorname{CCR}^{\mathrm{Weyl}}\left(\mathcal{Y}_{\mathbb{R}}\right)$ is $\mathbb{1}$, whereas in the case of $\operatorname{CCR}^{\text {reg }}\left(\mathcal{Y}_{\mathbb{R}}\right)$ we obtain a large gauge-invariant algebra.

7.1.2. Fock quantization of a charged symplectic dynamics. The concept of stability of dynamics in the charged case is analogous to the neutral case. 
Definition 7.2. We say that $\left(\mathcal{Y},(\cdot \mid \omega \cdot),(\cdot \mid \beta \cdot),\left(r_{t}\right)_{t \in \mathbb{R}}\right)$ is a weakly stable dynamics if the following conditions are true:

(1) $(\cdot \mid \beta \cdot)$ is a positive definite sesquilinear form. We equip $\mathcal{Y}$ with the norm $\|y\|_{\text {en }}:=$ $(y \mid \beta y)^{\frac{1}{2}}$. We denote by $\mathcal{Y}_{\text {en }}$ the completion of $\mathcal{Y}$ with respect to this norm.

(2) $t \mapsto r_{t}$ is bounded and strongly continuous. Thus we can extend $r_{t}$ to a strongly continuous group on $\mathcal{Y}_{\mathrm{en}}$ and define its generator $\mathrm{i} b$, so that $r_{t}=\mathrm{e}^{\mathrm{ti} b}$.

(3) $\operatorname{Ker} b=\{0\}$, or equivalently, $\bigcap_{t \in \mathbb{R}} \operatorname{Ker}\left(r_{t}-\mathbb{1}\right)=\{0\}$.

(4) $\mathcal{Y} \subset \operatorname{Dom} b$ and

$$
\left(y_{1} \mid \beta y_{2}\right):=\mathrm{i}\left(y_{1} \mid \omega b y_{2}\right), \quad y_{1}, y_{2} \in \mathcal{Y}
$$

If in addition

$$
\left|\left(y_{1} \mid \omega y_{2}\right)\right| \leq\left(y_{1} \mid \beta y_{1}\right)^{\frac{1}{2}}\left(y_{2} \mid \beta y_{2}\right)^{\frac{1}{2}}
$$

so that $(\cdot \mid \omega \cdot)$ can be extended to the whole $\mathcal{Y}_{\text {en }}$, we will say that the dynamics is strongly stable.

TheOrem 7.3. Let $\left(\mathcal{Y},(\cdot \mid \omega \cdot),(\cdot \mid \beta \cdot),\left(r_{t}\right)_{t \in \mathbb{R}}\right)$ be a weakly stable dynamics. Then

(1) $r_{t}$ are unitary transformations on the Hilbert space $\mathcal{Y}_{\mathrm{en}}$.

(2) $b$ is selfadjoint and $\operatorname{Ker} b=\{0\}$.

Set $q:=\operatorname{sgn} \mathrm{b}, \mathrm{j}:=\mathrm{i} \operatorname{sgn} \mathrm{b}$ and $h:=|b|$. Clearly $h$ is positive, and $r_{t}=\mathrm{e}^{\mathrm{tj} h}$.

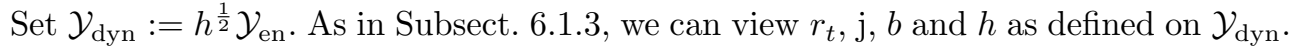
In what follows we drop the subscript dyn from $\mathcal{Y}_{\text {dyn }}$.

Let $\mathbb{1}_{ \pm}:=\mathbb{1}_{0, \infty[}( \pm b)=\mathbb{1}_{\{ \pm 1\}}(q), \mathcal{Y}_{ \pm}:=\operatorname{Ran} \mathbb{1}_{ \pm}$. Let $\mathcal{Z}$ denote the space $\mathcal{Y}$ equipped with the complex structure given by j. (In other words, $\mathcal{Z}:=\mathcal{Y}_{+} \oplus \overline{\mathcal{Y}}_{-}$).

The operators $h, q$ and $b$ preserve $\mathcal{Y}_{ \pm}$. Hence they can be viewed as complex linear operators on $\mathcal{Z}$ as well, in which case they will be denoted $h_{\mathcal{Z}}, q_{\mathcal{Z}}$ and $b_{\mathcal{Z}}$.

Consider the space $\Gamma_{\mathrm{s}}(\mathcal{Z})$. For $y \in \mathcal{Y}$, let us introduce the charged fields on $\mathcal{Y}$, which are closed operators on $\Gamma_{\mathrm{s}}(\mathcal{Z})$ defined by

$$
\psi^{*}(y)=a^{*}\left(\mathbb{1}_{+} y\right)+a\left(\overline{\mathbb{1}_{-} y}\right), \quad \psi(y)=a\left(\mathbb{1}_{+} y\right)+a^{*}\left(\overline{\mathbb{1}_{-} y}\right) .
$$

We obtain a charged CCR representation

$$
\mathcal{Y} \ni y \mapsto \psi(y) \in C l\left(\Gamma_{\mathrm{s}}(\mathcal{Z})\right) .
$$

Define the selfadjoint operators on $\Gamma_{\mathrm{s}}(\mathcal{Z})$ :

$$
H:=\mathrm{d} \Gamma\left(h_{\mathcal{Z}}\right), \quad Q:=\mathrm{d} \Gamma\left(q_{\mathcal{Z}}\right) .
$$

Clearly,

$$
\mathrm{e}^{\mathrm{i} t H} \psi(y) \mathrm{e}^{-\mathrm{i} t H}=\psi\left(\mathrm{e}^{\mathrm{i} t b} y\right), \quad \mathrm{e}^{\mathrm{i} \theta Q} \psi(y) \mathrm{e}^{-\mathrm{i} \theta Q}=\psi\left(\mathrm{e}^{\mathrm{i} \theta} y\right), \quad y \in \mathcal{Y} .
$$

DeFinition 7.4. 7.9 is called the positive energy Fock quantization for the dynamics $t \mapsto r_{t}$.

EXAMPLE 7.5. Let us describe a typical application of the charged bosonic formalism.

Let $\mathcal{Y}$ be the space of complex solutions of the Klein-Gordon equation that have a compact support on any space-like hypersurface. We obtain $\mathcal{Z}$ by switching the sign of the imaginary unit on negative frequency solutions. During quantization, this means that for negative frequency solutions we switch the role of creation and annihilation operators. 
As in the neutral case we can allow for a stationary globally hyperbolic space-time and a position-dependent mass. In addition, we can include a time-independent external vector potential.

\subsection{Charged fermionic systems}

7.2.1. Algebraic quantization of a unitary dynamics. Let $(\mathcal{Y},(\cdot \mid \cdot))$ be a complex Hilbert space describing a charged fermionic system. A strongly continuous 1-parameter group $\left\{r_{t}\right\}_{t \in \mathbb{R}}$ with $r_{t} \in U(\mathcal{Y})$ will be called a unitary dynamics.

Clearly, by taking the real scalar product $y_{1} \nu y_{2}:=\operatorname{Re}\left(y_{1} \mid y_{2}\right)$ we can view $\mathcal{Y}_{\mathbb{R}}$ as a real Hilbert space. We can associate to our system the field algebra $\operatorname{CAR}^{C^{*}}\left(\mathcal{Y}_{\mathbb{R}}\right)$ with distinguished elements $\psi(y)$. We can equip it with the automorphism group $\widehat{\mathrm{e}^{\mathrm{i} \theta}}$ and $\hat{r}_{t}$ defined by

$$
\widehat{\mathrm{e}^{\mathrm{i} \theta}}(\psi(y))=\psi\left(\mathrm{e}^{\mathrm{i} \theta} y\right), \quad \hat{r}_{t}(\psi(y))=\psi\left(r_{t} y\right)
$$

Similarly to the bosonic case, for the observable algebra we choose the so-called gaugeinvariant $C A R$ algebra $\operatorname{CAR}_{\text {gi }}^{C^{*}}(\mathcal{Y})$, which is defined as the set of elements of $\operatorname{CAR}^{C^{*}}\left(\mathcal{Y}_{\mathbb{R}}\right)$ fixed by $\widehat{\mathrm{e}^{\mathrm{i} \theta}}$. Note that $\operatorname{CAR}_{\text {gi }}^{C^{*}}(\mathcal{Y})$ is contained in the even algebra $\operatorname{CAR}_{0}^{C^{*}}\left(\mathcal{Y}_{\mathbb{R}}\right)$ and is preserved by the dynamics $\hat{r}_{t}$.

7.2.2. Fock quantization of a unitary dynamics. Let $\mathrm{i} b$ be the generator of $r_{t}$, so that $r_{t}=\mathrm{e}^{\mathrm{i} t b}$ and $b$ is selfadjoint.

DEFINITION 7.6. We say that the dynamics $t \mapsto r_{t} \in U(\mathcal{Y})$ is nondegenerate if

$$
\operatorname{Ker} b=\{0\} \text {, or equivalently } \bigcap_{t \in \mathbb{R}} \operatorname{Ker}\left(r_{t}-\mathbb{1}\right)=\{0\} \text {. }
$$

Set $q:=\operatorname{sgn} \mathrm{b}, \mathrm{j}:=\mathrm{i} \operatorname{sgn} \mathrm{b}$ and $h:=|b|$. Clearly $h$ is positive, and $r_{t}=\mathrm{e}^{t \mathrm{j} h}$. Let $\mathbb{1}_{ \pm}:=\mathbb{1}_{] 0, \infty[}( \pm b)=\mathbb{1}_{\{ \pm 1\}}(q), \mathcal{Y}_{ \pm}:=\operatorname{Ran} \mathbb{1}_{ \pm}$. Let $\mathcal{Z}$ denote the space $\mathcal{Y}$ equipped with the complex structure given by j. (In other words, $\mathcal{Z}:=\mathcal{Y}_{+} \oplus \overline{\mathcal{Y}}_{-}$).

The operators $h, q$ and $b$ preserve $\mathcal{Y}_{ \pm}$. Hence they can also be viewed as complex linear operators on $\mathcal{Z}$ as well, in which case they will be denoted $h_{\mathcal{Z}}, q_{\mathcal{Z}}$ and $b_{\mathcal{Z}}$.

Consider the space $\Gamma_{\mathrm{a}}(\mathcal{Z})$. For $y \in \mathcal{Y}$, let us introduce the charged fields on $\mathcal{Y}$, which are closed operators on $\Gamma_{\mathrm{a}}(\mathcal{Z})$

$$
\begin{aligned}
\psi^{*}(y) & =a^{*}\left(\mathbb{1}_{+} y\right)+a\left(\overline{\mathbb{1}_{-} y}\right), \\
\psi(y) & =a\left(\mathbb{1}_{+} y\right)+a^{*}\left(\overline{\mathbb{1}_{-} y}\right) .
\end{aligned}
$$

We obtain a charged CAR representation

$$
\mathcal{Y} \ni y \mapsto \psi(y) \in B\left(\Gamma_{\mathrm{a}}(\mathcal{Z})\right) .
$$

Define the selfadjoint operators on $\Gamma_{\mathrm{a}}(\mathcal{Z})$

$$
H:=\mathrm{d} \Gamma\left(h_{\mathcal{Z}}\right), \quad Q:=\mathrm{d} \Gamma\left(q_{\mathcal{Z}}\right) .
$$

Clearly,

$$
\mathrm{e}^{\mathrm{i} t H} \psi(y) \mathrm{e}^{-\mathrm{i} t H}=\psi\left(\mathrm{e}^{\mathrm{i} t b} y\right), \quad \mathrm{e}^{\mathrm{i} \theta Q} \psi(y) \mathrm{e}^{-\mathrm{i} \theta Q}=\psi\left(\mathrm{e}^{\mathrm{i} \theta} y\right), \quad y \in \mathcal{Y} .
$$

Definition 7.7. 7.13 is called the positive energy Fock quantization for the dynamics $t \mapsto r_{t}$. 
EXAMPLE 7.8. Let us describe a typical application of the charged fermionic formalism.

Let $\mathcal{Y}$ be the space of solutions of the Dirac equation that have a compact support on any space-like hypersurface. We obtain $\mathcal{Z}$ by switching the sign of the imaginary unit on negative frequency solutions. During quantization, this means that for negative frequency solutions we switch the role of creation and annihilation operators.

As in the neutral case we can allow for a stationary globally hyperbolic space-time and a position-dependent mass. In addition, we can include a time-independent external vector potential.

\subsection{Charge reversal}

7.3.1. Algebraic quantization of charge reversal. Let $(\mathcal{Y},(\cdot \mid \omega \cdot))$ be a charged symplectic space in the bosonic case, or let $(\mathcal{Y},(\cdot \mid \cdot))$ be a complex Hilbert space in the fermionic case.

Definition 7.9. We say that $\chi$ is a charge reversal iff $\chi$ is antilinear, $\chi^{2}=1$ or $\chi^{2}=-1$, and

$$
\begin{aligned}
\left(\chi y_{1} \mid \omega \chi y_{2}\right) & =\overline{\left(y_{1} \mid \omega y_{2}\right)}, \quad(\chi \text { is anticharged symplectic }) \text { in the bosonic case; } \\
\left(\chi y_{1} \mid \chi y_{2}\right) & =\overline{\left(y_{1} \mid y_{2}\right)}, \quad(\chi \text { is antiunitary }) \text { in the fermionic case. }
\end{aligned}
$$

Definition 7.10. Suppose that $\left\{r_{t}\right\}_{t \in \mathbb{R}}$ is a charged symplectic (resp. unitary) dynamics. We say that the dynamics is invariant under the charge reversal $\chi$ if

$$
\chi r_{t}=r_{t} \chi, \quad t \in \mathbb{R} .
$$

Similarly, if we have a group of symmetries $\left\{r_{g}\right\}_{g \in G}$ we say that it is invariant under charge reversal $\chi$ iff $r_{g} \chi=\chi r_{g}, g \in G$. by

In the bosonic case we define the (linear) automorphism $\hat{\chi}$ of the algebra $\operatorname{CCR}^{\text {reg }}\left(\mathcal{Y}_{\mathbb{R}}\right)$

$$
\hat{\chi}\left(\mathrm{e}^{\mathrm{i} \psi(y)+\mathrm{i} \psi^{*}(y)}\right)=\mathrm{e}^{\mathrm{i} \psi(\chi y)+\mathrm{i} \psi^{*}(\chi y)} .
$$

It restricts to an automorphism of $\mathrm{CCR}_{\mathrm{gi}}^{\mathrm{reg}}(\mathcal{Y})$.

In the fermionic case we define the (linear) automorphism $\hat{\chi}$ of the algebra $\operatorname{CAR}^{C^{*}}\left(\mathcal{Y}_{\mathbb{R}}\right)$ by $\hat{\chi}\left(\psi^{*}(y)\right)=\psi(\chi y)$. It restricts to an automorphism of $\operatorname{CAR}_{\mathrm{gi}}^{C^{*}}(\mathcal{Y})$.

7.3.2. Fock quantization of charge reversal. In the bosonic case, assume that the dynamics is weakly stable. In the fermionic case assume it is nondegenerate. Let $b, h, q$ etc. be constructed as before. In both bosonic and fermionic cases, it follows that

$$
\chi h=h \chi, \quad \chi b=-b \chi, \quad \chi q=-q \chi, \quad \chi \mathrm{j}=\mathrm{j} \chi .
$$

We denote $\chi_{\mathcal{Z}}$ the map $\chi$ considered on $\mathcal{Z}$. Note that $\chi_{\mathcal{Z}}$, unlike $\chi$, is unitary. We second-quantize it by the unitary $C:=\Gamma\left(\chi_{\mathcal{Z}}\right)$. We have

$$
C H C^{-1}=H, \quad C Q C^{-1}=-Q, \quad C \psi^{*}(y) C^{-1}=\psi(\chi y) .
$$

Note that

$$
C^{2}=1 \quad \text { or } \quad C^{2}=I .
$$


7.3.3. Neutral subspace. Assume that $\chi^{2}=1$. We can then define the space $\mathcal{Y}_{\chi}:=$ $\{y \in \mathcal{Y}: y=\chi y\}$ and restrict the dynamics and the symmetry group to $\mathcal{Y}_{\chi}$. One can call $\mathcal{Y}_{\chi}$ the neutral subspace of $\mathcal{Y}$. (In the fermionic case it is also called the Majorana subspace). Note that $\mathcal{Y}=\mathcal{Y}_{\chi} \oplus \mathrm{i} \mathcal{Y}_{\chi}$, hence the system can be viewed as a couple of neutral systems.

Let us describe the converse construction. Suppose that we have a neutral system $(\mathcal{Y}, \omega)$ or $(\mathcal{Y}, \nu)$ equipped with the dynamics $t \mapsto r_{t}$. We can extend it to a charged system as follows. We consider the complexified space $\mathbb{C} \mathcal{Y}$ equipped with the natural conjugation denoted by the "bar". We equip it with the antihermitian form, resp. scalar product

$$
\begin{aligned}
\left(y_{1} \mid \omega y_{2}\right) & :=\bar{y}_{1} \omega y_{2}, \\
\text { or }\left(y_{1} \mid y_{2}\right) & :=\bar{y}_{1} \nu y_{2}, \quad y_{1}, y_{2} \in \mathbb{C} \mathcal{Y} .
\end{aligned}
$$

We extend the dynamics $r_{t}$ to $\left(r_{t}\right)_{\mathbb{C}}$ on $\mathbb{C} \mathcal{Y}$. Clearly, $\left(r_{t}\right)_{\mathbb{C}}$ is a charged symplectic, resp. unitary dynamics and the complex conjugation $\chi y:=\bar{y}$ is a charge reversal satisfying $\chi^{2}=1$. One gets back the original system by the restriction to the neutral subspace.

\subsection{Time reversal in charged systems}

7.4.1. Algebraic quantization of time reversal. Let $(\mathcal{Y},(\cdot \mid \omega \cdot))$ be a charged symplectic space in the bosonic case, or let $(\mathcal{Y},(\cdot \mid \cdot))$ be a complex Hilbert space in the fermionic case.

Definition 7.11. We say that $\tau \in L(\mathcal{Y})$ is a time reversal iff $\tau r_{t}=r_{-t} \tau, \tau$ is antilinear, $\tau^{2}=\mathbb{1}$ or $\tau^{2}=-1$, and

$\left(\tau y_{1} \mid \omega \tau y_{2}\right)=-\overline{\left(y_{1} \mid \omega y_{2}\right)}, \quad(\tau$ is anticharged antisymplectic) in the bosonic case;

$\left(\tau y_{1} \mid \tau y_{2}\right)=\overline{\left(y_{1} \mid y_{2}\right)}, \quad(\tau$ is antiunitary $)$ in the fermionic case.

In the bosonic case we define the antilinear *-automorphism $\hat{\tau}$ of the algebra $\operatorname{CCR}^{\mathrm{reg}}\left(\mathcal{Y}_{\mathbb{R}}\right)$ by

$$
\hat{\tau}\left(\mathrm{e}^{\mathrm{i} \psi(y)+\mathrm{i} \psi^{*}(y)}\right)=\mathrm{e}^{-\mathrm{i} \psi(\tau y)-\mathrm{i} \psi^{*}(\tau y)} .
$$

It restricts to an antilinear $*$-automorphism of $\operatorname{CCR}_{\mathrm{gi}}^{\mathrm{reg}}(\mathcal{Y})$.

In the fermionic case we define the antilinear $*$-automorphism $\hat{\tau}$ of $\operatorname{CAR}^{C^{*}}\left(\mathcal{Y}_{\mathbb{R}}\right)$ by $\hat{\tau}(\psi(y))=\psi(\tau y)$. It restricts to an automorphism of $\operatorname{CAR}_{\mathrm{gi}}^{C^{*}}(\mathcal{Y})$.

7.4.2. Fock quantization of time reversal. Clearly, we have $\tau q=q \tau$. Thus $\tau \mathcal{Y}_{+}=\mathcal{Y}_{+}$, $\tau \mathcal{Y}_{-}=\mathcal{Y}_{-}$.

Let $\tau_{\mathcal{Z}}$ denote $\tau$ considered on $\mathcal{Z}$. It is antilinear. We second-quantize $\tau$ by the antiunitary $T:=\Gamma(\tau)$. We obtain

$$
\begin{aligned}
T_{H T^{-1}}=H, & T \mathrm{e}^{\mathrm{i} t H} T^{-1}=\mathrm{e}^{-\mathrm{i} t H}, \\
T Q T^{-1}=Q, & T \mathrm{e}^{\mathrm{i} \theta Q} T^{-1}=\mathrm{e}^{-\mathrm{i} \theta Q}, \\
T \psi(y) T^{-1}=\psi(\tau y), & T \psi^{*}(y) T^{-1}=\psi^{*}(\tau y), \\
T^{2}=\mathbb{1} \quad \text { or } & T^{2}=I .
\end{aligned}
$$


7.4.3. Commutation between charge and time reversal. It is natural to assume that on the observable algebra $(\hat{\chi} \hat{\tau})^{2}$ is the identity. This is guaranteed if $(\chi \tau)^{2}$ equals $\mathbb{1}$ or $-\mathbb{1}$. This leads to the following (anti)commutation relations for $\chi$ and $\tau$ :

$$
\tau \chi=\chi \tau \quad \text { or } \quad \tau \chi=-\chi \tau .
$$

However, we are free to multiply either $\chi$ or $\tau$ by i. Therefore, possibly after a redefinition of $\chi$ or $\tau$, we can always assume that

$$
\tau \chi=\chi \tau .
$$

Thus we have 3 commuting symmetries: $\chi, \tau$ and $\chi \tau$. They satisfy one of the following sets of relations:

$$
\begin{array}{rrrr}
\chi^{2}=1, & \tau^{2}=11, & (\chi \tau)^{2}=11 \\
\chi^{2}=-11, & \tau^{2}=-1, & (\chi \tau)^{2}=1 ; \\
\chi^{2}=11, & \tau^{2}=-11, & (\chi \tau)^{2}=-11 \\
\chi^{2}=-11, & \tau^{2}=11, & (\chi \tau)^{2}=-11 .
\end{array}
$$

Acknowledgments. The research of J. Dereziński is supported in part by the grant N N201 270135.

\section{References}

[Ar1] H. Araki, On quasi-free states of CAR and Bogolubov automorphism, Publ. RIMS Kyoto Univ. 6 (1970) 385-442.

[Ar2] H. Araki, On quasi-free states of canonical commutation relations II, Publ. RIMS Kyoto Univ. 7 (1971/72), 121-152.

[Ar3] H. Araki, Bogoliubov automorphisms and Fock representations of canonical anticommutation relations, in: Contemp. Math. 62 (1987), 23.

[ArShi] H. Araki and M. Shiraishi, On quasi-free states of canonical commutation relations I, Publ. RIMS Kyoto Univ. 7 (1971/72), 105-120.

[BSZ] J. C. Baez, I. E. Segal and Z. Zhou, Introduction to Algebraic and Constructive Quantum Field Theory, Princeton NJ, Princeton University Press, 1991.

[BR] O. Bratteli and D. W. Robinson, Operator Algebras and Quantum Statistical Mechanics Vols I, II, Springer, Berlin, 1981.

[CMR] M. Courbage, S. Miracle-Sole, D. W. Robinson, Normal states and representations of the canonical commutation relations, Ann. I.H.P. 14 (1971), 171-178.

[De] J. Dereziński, Introduction to representations of canonical commutation and anticommutation relations, in: Large Coulomb Systems - QED, J. Dereziński and H. Siedentop (eds.), Lecture Notes in Physics 695, Springer, 2006.

[DG1] J. Dereziński and C. Gérard, Asymptotic completeness in quantum field theory. Massive Pauli-Fierz Hamiltonians, Reviews in Mathematical Physics 11 (1999), 383-450.

[DG2] J. Dereziński and C. Gérard, Mathematics of quantization and quantum fields, in preparation.

[Di1] P. A. M. Dirac, The quantum theory of the emission and absorption of radiation, Proc. Royal Soc. London Series A 114 (1927), 243. 
[Em] G. Emch, Algebraic Methods in Statistical Mechanics and Quantum Field Theory, Wiley-Interscience, 1972.

[Fo] V. Fock, Konfigurationsraum und zweite Quantelung, Z. Phys. 75 (1932), 622-647.

[JW] P. Jordan and E. Wigner, Pauli's equivalence prohibition, Zeitschr. Phys. 47 (1928), 631.

[GJ] J. Glimm and A. Jaffe, Quantum Physics. A Functional Integral Point of View, second edition, Springer, New York, 1987.

[Ha] R. Haag, Local Quantum Physics, New York, Springer, 1993.

[PR] R. J. Plymen and P. L. Robinson, Spinors in Hilbert Spaces, Cambridge University Press, 1994.

[RS1] M. Reed and B. Simon, Methods of Modern Mathematical Physics, I. Functional Analysis, London, Academic Press, 1980.

[RS2] M. Reed and B. Simon, Methods of Modern Mathematical Physics, II. Fourier Analysis, Self-Adjointness, London, Academic Press, 1975.

[Si] B. Simon, The $P(\phi)_{2}$ Euclidean (Quantum) Field Theory, Princeton University Press, 1974.

[Sla] J. Slawny, On factor representations of the $C^{*}$-algebra of canonical commutation relations, Comm. Math. Phys. 24 (1971), 151-170.

[Sr] M. Srednicki, Quantum Field Theory, Cambridge University Press, 2007.

[We] S. Weinberg, The Quantum Theory of Fields I, Cambridge University Press, 2005. 